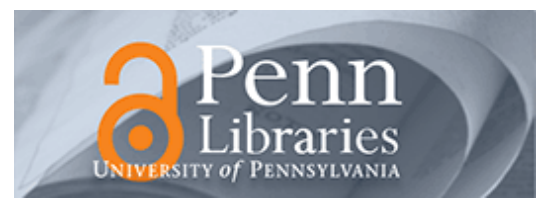

University of Pennsylvania

ScholarlyCommons

Marketing Papers

Wharton Faculty Research

$3-2014$

\title{
Entry- and Sunk-Cost Spillovers from the Rival: Evidence from Entry and Expansion of KFC and McDonald's in Chinese Cities
}

Aamir R. Hashmi

Ping Xiao

Qiaowei Shen

University of Pennsylvania

Follow this and additional works at: https://repository.upenn.edu/marketing_papers

Part of the Business Administration, Management, and Operations Commons, Business Analytics Commons, Business Intelligence Commons, International Business Commons, Management Sciences and Quantitative Methods Commons, Marketing Commons, and the Strategic Management Policy Commons

\section{Recommended Citation}

Hashmi, A. R., Xiao, P., \& Shen, Q. (2014). Entry- and Sunk-Cost Spillovers from the Rival: Evidence from Entry and Expansion of KFC and McDonald's in Chinese Cities. http://dx.doi.org/10.2139/ssrn.2410700

This is an unpublished manuscript.

This paper is posted at ScholarlyCommons. https://repository.upenn.edu/marketing_papers/355

For more information, please contact repository@pobox.upenn.edu. 


\title{
Entry- and Sunk-Cost Spillovers from the Rival: Evidence from Entry and Expansion of KFC and McDonald's in Chinese Cities
}

\begin{abstract}
We model the entry and expansion of KFC and McDonald's in Chinese cities as a dynamic game. We assume that the observed entry and expansion decisions are equilibrium outcomes. This allows us to recover the structural parameters of the game without solving for equilibrium. We use the estimated model to study the entry- and sunk-cost spillovers from the rival. Our estimates suggest substantial spillovers to the cost of entering a new city. For example, if the rival is not present in the city in which the chain is entering and the distance from the nearest city where the rival is present decreases by 100 kilometers, the cost of entry into the city decreases by 1.22 standard deviations for KFC and 1.52 standard deviations for McDonald's. If the rival is already present in the city, a one-unit increase in the number of rival's outlets decreases the cost of entry by 0.59 standard deviations for McDonald's but increases it by 1.49 standard deviations for KFC. We also find that the spillovers to the sunk cost of opening a new outlet are much smaller. Hence the expansion within a city is not as much influenced by the presence of the rival as is the entry into a new city.
\end{abstract}

\section{Keywords}

dynamic games, dynamic entry, spillovers, fastfood industry, China

\section{Disciplines}

Business | Business Administration, Management, and Operations | Business Analytics | Business Intelligence | International Business | Management Sciences and Quantitative Methods | Marketing | Strategic Management Policy

\section{Comments}

This is an unpublished manuscript. 


\section{Entry- and Sunk-Cost Spillovers from the Rival: Evidence from Entry and Expansion of KFC and McDonald's in Chinese Cities}

\author{
Aamir Rafique HASHMI \\ Department of Economics \\ National University of Singapore
}

\author{
Ping XIAO \\ Department of Marketing \\ NUS Business School
}

National University of Singapore

Qiaowei SHEN

Marketing Department

The Wharton School

University of Pennsylvania

March 18, 2014 


\begin{abstract}
We model the entry and expansion of KFC and McDonald's in Chinese cities as a dynamic game. We assume that the observed entry and expansion decisions are equilibrium outcomes. This allows us to recover the structural parameters of the game without solving for equilibrium. We use the estimated model to study the entry- and sunk-cost spillovers from the rival. Our estimates suggest substantial spillovers to the cost of entering a new city. For example, if the rival is not present in the city in which the chain is entering and the distance from the nearest city where the rival is present decreases by 100 kilometers, the cost of entry into the city decreases by 1.22 standard deviations for KFC and 1.52 standard deviations for McDonald's. If the rival is already present in the city, a one-unit increase in the number of rival's outlets decreases the cost of entry by 0.59 standard deviations for McDonald's but increases it by 1.49 standard deviations for KFC. We also find that the spillovers to the sunk cost of opening a new outlet are much smaller. Hence the expansion within a city is not as much influenced by the presence of the rival as is the entry into a new city.
\end{abstract}

Keywords: Dynamic Games; Dynamic Entry ; Fastfood Industry; China

JEL Codes: L13; L81; M31 


\section{Introduction}

The decisions about market selection are at the heart of the growth strategy of any business 1 In this paper, we study the empirical relationship between market structure and, entry and expansion decisions of retail chains. We define market structure broadly to include a chain's own network of outlets, its rivals' network of outlets and some other market-specific characteristics. Our primary objective is to study how the rivals' presence in and around a market affects a chain's entry and expansion decisions 2 Our empirical setting is the entry and expansion of Western fastfood chains in various Chinese cities $3^{3}$ over a period of two decades. Due to the lack of data on the demand side, we focus on spillovers to the cost of entering a new market and to the sunk cost of expansion within an existing market.

First consider a chain's decision to enter a new city. If we take the demand side as given, the rivals' presence in or around the city may increase or decrease the chain's perceived cost of entry. Next consider a chain's decision to expand within a city by opening more outlets. Once again taking the demand side as given, the rivals' presence may increase or decrease the the sunk cost of opening new outlets. The entry and sunk costs are economic costs and not directly observable. We need a structural model to recover them from the data. If we model these costs as functions of the size and the density of the rivals' network of outlets, we can quantify the cost-side spillovers from the rival. This is the approach that we take in this paper 4

The spillovers from the rivals are not the only consideration when a multi-outlet chain contemplates entry into a new market or expansion within an existing market. The spillovers from own network of outlets are equally, or perhaps more, important. When a chain considers entry into a new market, the proximity of the market to the chain's own existing network of outlets is likely to reduce the cost of entry due to the economies of density. Once in the market, further expansion may also benefit from own presence in the market. On top of the effects from own and rivals' networks, market-specific charateristics also play an important role in entry and expansion decisions. These characteristics may include market demographics and per capita income of the consumers.

We attempt to quantify these multiple effects in the context of entry and expansion of

\footnotetext{
${ }^{1}$ In a recent video interview, McKinsey \& Company's Chris Bradly (a principal in McKinsey's Sydney office) said: "If you look at what drives the growth of companies, it turns out that selection at a micromarket level is much more important than trying to gain market share. In fact, 80 percent of growth is explained by decisions about where to compete or by market selection." [The video interview is available at http: //www.mckinsey.com/insights/strategy/the_art_of_strategy (last accessed: November 2, 2013)]

${ }^{2}$ In this paper, 'entry' refers to the decision of opening the first outlet in a market and 'expansion' refers to the decision of adding more outlets to a market conditional on entry.

${ }^{3}$ We define market to be a city and hence use the words "market" and "city" interchangeably.

${ }^{4}$ Two important caveats apply: 1) although our approach allows us to recover the net spillovers from the rivals, it cannot disentangle the positive and negative spillovers; 2) although our model allows us to quantify the entry and sunk costs it cannot explain what exactly these costs are and how the rivals' presence actually affects them.
} 
two major Western fastfood chains, Kentucky Fried Chicken (KFC) and McDonald's, into various Chinese cities. The rapid expansion of the two chains into numerous Chinese cities within the last couple of decades provides a unique opportunity to study their entry and expansion decisions in a strategic environment. Because entry and expansion are inherently dynamic decisions, we specify and estimate a dynamic game of entry and expansion to understand these decisions 5 In our model, every period, for each market, the two chains simultaneously decide whether to enter the market (if they are not in yet) or to expand the number of outlets in the market. Their decisions depend on: 1) their overall expansion policy in the country; 2) their own network of outlets in the market and in the neighboring markets; 3) their rival's network in the market and in the neighboring markets; 4) various characteristics of the market; 5) their expectations of their rival's entry and expansion decisions; and 6) their expectations about the future evolution of the market characteristics.

The estimation of such a large scale game was almost inconceivable until very recently. The full-solution methods, like Rust's Nested-Fixed-Point Algorithm [Rust (1987)], are not feasible for a game of this scale because they require computation of equilibrium at every iteration of the estimation process. For a game like the one that we specify in this paper, the enormity of the state space makes it practically impossible to compute equilibrium even once ${ }^{6}$ Recently a number of authors have proposed methods to estimate dynamic games without computing equilibrium. In this paper we use the method proposed by Bajari et al. (2007). The principal advantage of their approach is its relatively lower computational burden.

We find evidence of substantial spillovers from the rival to the cost of entering a new city. For example, if the rival is not present in the city in which the chain is entering and the distance from the nearest city where the rival is present decreases by 100 kilometers, the cost of entry into the city decreases by 1.22 standard deviations for KFC and 1.52 standard deviations for McDonald's. If the rival is already present in the city, a one-unit increase in the number of rival's outlets decreases the cost of entry by 0.51 standard deviations for McDonald's but increases it by 1.49 standard deviations for KFC.

We also find that the spillovers to the sunk cost of opening a new outlet are much smaller. For example, a 10-unit increase in the rival's network density (to be defined later) decreases the sunk cost by 0.03 standard deviations for KFC and increases it by 0.03 stan-

\footnotetext{
${ }^{5}$ Entry into a new city is a dynamic decision because a chain can either enter now or wait until the next period. If it enters now, it will have to pay the cost of entry and will start receiving profits from the new outlets in the next period. If it waits, it will face the same choice, enter or wait, next period.

Similarly, expansion within a city is also a dynamic decision because a chain can either expand now or wait until the next period. If it expands now, it will have to pay the sunk cost for every new outlet that it opens and it will start getting the profits from the new outlets next period. If it decides to wait, it will face the same choice, expand or wait, next period.

${ }^{6}$ There are 11 state variables for each chain in our model (see Section 3 for details). These include 2 privately observed cost shocks. Even if we assumed just 5 possible values for each of these shocks, the number of states in the state space would still be a whopping $9.29 \mathrm{E}+15$.
} 
dard deviations for McDonald's. Similarly, the number of rival's outlets in the city also has a small effect on the sunk cost: a one-unit increase in the rival's number of outlets decreases the sunk cost by 0.01 standard deviations for both KFC and McDonald's. Hence the expansion within a city is not as much influenced by the presence of the rival as is the entry into a new city.

This paper connects to a number of literatures. Firstly, it is closely related to the literature on empirical models of entry Berry and Reiss (2007)]. The pioneering papers in this literature are Bresnahan and Reiss (1991) and Berry (1992). Mazzeo (2002), Seim (2006) and Zhu et al. (2009) extend the framework to endogenously consider product differentiation along with entry decisions in static game-theoretic environments. We contribute to this literature by using a dynamic-game framework to study the relationship between market structure and the firms' entry and expansion decisions.

Secondly, and most importantly, it is related to the empirical literature on retail chain entry and expansion. This literature can be classified into three groups depending on whether the underlying model is static or dynamic and whether it allows for strategic interactions among the firms.

The first group includes papers that model entry as a static game. Notable papers in this group are: Toivanen and Waterson (2005), Jia (2008) and Nishida (2013). Toivanen and Waterson (2005) allow for a positive effect from the rival in a chain's market entry decision using data from the United Kingdom's fastfood industry. They model the duoploy between Burger King and McDonald's as a static sequential game. Their structural estimates suggest that a rival's presence in the market leads a firm to expect a larger market. They argue that the spillover effect mainly operates through learning. Jia (2008) studies the positive spillover effects when locating multiple stores in nearby regions in the context of WalMart's market entry decisions. Her modelling strategy involves a static three-stage game. Nishida (2013) extends Jia's approach to consider the decision of how many stores to open in a chain's entry decision using data from the convenience-store industry of Japan, but constrains the spillover effect to be positive as in Jia (2008). The aforementioned papers build on game-theoretic models of static entry.

The second group consists of papers that employ single-agent dynamic models to study a chain's location and expansion decisions. Notable contributions in this vein of literature are Holmes (2011) and Toivanen and Waterson (2011). Holmes (2011) estimates the economies of density in the store location decisions of Wal-Mart in the United States. He considers cannibalization of sales by nearby stores of the same chain but does not model competition effect from other retail chains. He finds that the economies of density are substantial and extend beyond the savings in trucking costs. Toivanen and Waterson (2011) study the expansion of McDonald's in the United Kingdom up to 1990, a period when it can reasonably be considered a monopoly in the UK market. They find positive cannibalization effect from own outlets on the demand side but economies of density on the cost side. 
The third group includes papers that model entry and expansion as dynamic games. Important contributions to this strand of literature are Aguirregabiria and Magesan (2012) and Yang (2012). Aguirregabiria and Magesan (2012) use the dataset in Toivanen and Waterson (2005). Their main empirical contribution is to show that when a chain's beliefs about its rival's strategy are biased, the chain runs the risk of underestimating the potential competition effects from the rival after its own entry. Yang (2012) studies the effect of information spillovers from incumbent rivals on a chain's decision to enter a new market. Prior to entry, potential entrants are uncertain about the size of the market that they are about to enter. The past entry and exit decisions of incumbent rivals reduce this uncertainty and allow the potential entrants to make more informed entry decisions. The present paper is closest to Yang (2012) as both papers estimate dynamic games to quantify the spillovers from rivals. However, this paper differs from his in a number of ways. First, we focus on entry and expansion decisions of the chains while his focus is on binary entry and exit decisions. Second, we allow for multiple spillover effects within the chain and across the chains while he focuses solely on information spillovers from rivals. Third, we explicitly model the effect of own network on entry while he abstracts away from this effect. Fourth, our empirical setting is the fastfood market in China while he studies the Canadian fastfood market.

Thirdly and finally, this paper provides yet another application of the recent methods to estimate dynamic games. The leading methodology papers in this literature are Aguirregabiria and Mira (2007), Bajari et al. (2007), Pakes et al. (2007) and Pesendorfer and Schmidt-Dengler (2008). Ackerberg et al. (2007) provide a comparison of these methods.

We have organized the rest of the paper as follows. In Section 2 we provide an overview of the Chinese fastfood industry and introduce our dataset. In Section 3 we present our model. In Section 4 we discuss our estimation strategy. We report the estimation results in Section 5. We discuss the evidence on spillovers in Section 6 and conclude in Section 7.

\section{Preliminaries}

In this section we provide an overview of the history of Western chains in the Chinese fastfood market and introduce our dataset. The discussion in this section draws mainly on Shen and Xiao (2013).

\subsection{Industry Overview}

The history of Western fastfood in China began in November 1987 when KFC opened its first outlet in Beijing. Three years later, in October 1990, McDonald's opened its first outlet in Shenzhen and the competition between the two formally started in the world's largest emerging market. 
In Figure 1 we plot the total number of outlets and their growth rates in China for the two chains during the period from 1990 to 2007. Although globally McDonald's is far ahead of $\mathrm{KFC}$ in terms of number of outlets, their positions are reversed in the Chinese market. Not only did KFC enter the country first, its expansion has also been much faster than that of McDonald's. By the end of 2007, KFC had close to two thousand outlets in China whereas McDonald's had slightly more than a thousand.

In Table 1 we report, for selected years, the total number of outlets together with the number of cities entered by each chain. The first thing to note is that although KFC has been ahead of McDonald's throughout the period, it has extended its lead very sharply since 2000. The second thing to note is that most of the KFC's lead is due to its entry in more cities. By 2007, McDonald's had entered 142 cities (out of the total 246 in our sample) whereas KFC was present in 230 cities, a lead of $62 \%$. If we compare within city density of outlets, McDonald's had 7.1 outlets per city while KFC had 8.4, a lead of $18 \%$. The third thing to note is that the scale of entry and expansion varies over the sample period. This variation will be helpful to identify the parameters of interest.

Table 2, in which we report statistics about the first mover in each city, provides further evidence that KFC has been much more aggressive than McDonald's in entering new cities. By the end of 2007, at least one of the two chains was present in 236 cities. KFC was the first mover in $177(75 \%)$ of these cities and McDonald's in 33 (14\%). In the remaining 26 $(11 \%)$ cities, both chains entered in the same calendar year. On the average, McDonald's waited 3.5 years to enter a city where KFC was already present.

We model the entry and expansion of the Western fastfood chains in the Chinese market over the 1990-2007 period as a duopoly game between KFC and McDonald's:7 Although lately some other Western fastfood chains have entered the Chinese market, their presence is still modest by the end of our sample period $]^{8}$ Similarly, none of the local Chinese fastfood chains is large enough to be considered a strategic player in the national fastfood market. Also, the Western fastfood is quite distinct from the traditional Chinese fastfood and hence the two are not likely to be close substitutes.

We do not distinguish between the franchised and non-franchised outlets. There are at least two reasons for doing so. First, during our sample period the franchised outlets accounted for a small percentage of the total for both chains 99 Second, our focus in this

\footnotetext{
${ }^{7}$ It is a popular perception that KFC and McDonald's are close rivals in the Chinese market. For example, The Wall Street Journal Asia reported on February 29, 2012: "McDonald's Corp. is launching a new ad campaign in China ... to win market share from its dominant rival, Yum Brands Inc.'s KFC." [p. 19]

${ }^{8}$ For example, Subway entered the Chinese market in 1995 but had only around 150 outlets by the end of 2007. Burger King entered in 2005 and had fewer than 50 outlets by the end of 2007 .

${ }^{9} \mathrm{KFC}$ opened its first franchised outlet in 2000 and by the end of 2005 it had only 37 franchised outlets, which were $2.5 \%$ of its then total stock of 1462 outlets. (http://franchise.business-opportunities. biz/2006/04/21/kfc-makes-it-easier-to-buy-a-franchise [last accessed: October 7, 2013]) McDonald's launched a pilot franchise program in 2004 and had only 6 franchised outlets by early 2010. (http://www. reuters.com/article/2010/05/06/us-mcdonalds-china-idUSTRE6451W420100506 [last accessed: March 1,
} 
paper is on entry and expansion decisions. These decisions are made by the top management of the chains at the national level and franchisees have little say in them.

\subsection{Data}

Our basic unit of analysis is a city. Our sample consists of 246 Chinese cities spanning an eighteen-year period from 1990 to 2007 10 Our dataset includes information on gross domestic product (GDP), population and area of these cities. We have also gathered information on geographic coordinates of the cities. This enables us to calculate the geographic distance between any possible pair of cities in our sample. We track entry and expansion of KFC and McDonald's in these cities for the entire sample period 11 In the rest of this sub-section we highlight some important features of the dataset.

In Table 3 we show the frequency distribution of the number of outlets at the end of the sample period. There are 16 cities (6.5\% of total) in the sample in which KFC has yet to enter and 104 cities (42.3\%) in which McDonald's has yet to enter. The number of cities with 1 or 2 KFC outlets is 131 (53.3\%) and with 1 or 2 McDonald's outlets it is 89 (36.2\%). There are also a number of cities with a sizable presence by both chains. For example, there are 39 cities $(15.8 \%)$ with 11 or more KFC outlets and 23 cities (9.4\%) with 11 or more McDonald's outlets.

In the upper panel of Table 4 we show the frequency distribution of the number of outlets for the entire sample. This information is important because the number of outlets in a city is a key state variable in our model. There are many zeros in the data. Out of a total number of 4, 428 observations (246 cities times 18 years), there are 3,168 (71.5\%) observations with zero outlets for KFC and 3,638 (82.2\%) observations with zero outlets for McDonald's. The number of observations with 1 or 2 outlets is 755 (17.1\%) for KFC and $466(10.5 \%)$ for McDonald's. The number of observations with 11 or more outlets is just $164(3.7 \%)$ for KFC and $116(2.6 \%)$ for McDonald's.

In the lower panel of Table 4 we show the frequency distribution of the new outlets opened during a year. This is important because the choice of new outlets is the only decision variable in our model ${ }^{12}$ Here again we see lots of zeros. There are 3,643 $(82.3 \%)$ observations with zero new outlets for KFC and 4,018 (90.7\%) such observations for McDonald's. When a chain does open new outlets, most of the time it opens either 1 or 2 . There are $594(13.5 \%)$ observations with 1 or 2 new outlets for KFC and $295(6.7 \%)$ such observations for McDonald's. The rest of the observations (191 or 4.3\% for KFC and 115

${ }^{10}$ According to the World Bank, total population of China was 1,318 million in 2007. Out of these, $45 \%$ or 593.1 million people lived in urban areas. Total population of the 246 cities in our sample was 336.5 million in 2007. This amounts to $56.7 \%$ of total urban population in China in that year.

${ }^{11}$ For details on collection and construction of the dataset see Shen and Xiao (2013).

${ }^{12}$ We do not model entry and expansion decisions separately. Instead, we write a unified decision problem that encompasses the two decisions. See Section 3 for details.
} 
or $2.6 \%$ for McDonald's) are with 3 or more new outlets.

In Table 5 we report summary statistics for a number of variables. Some of these variables are chain-specific and some city-specific. The first chain-specific variable is the total number of outlets of a chain in a city at the beginning of a calendar year. The average number of outlets is 1.8 for KFC and 1.2 for McDonald's. As we saw in Table 4 , there is a lot of variation in the number of outlets. This is reflected in high standard deviations (SDs) of the number of outlets: 8.8 for KFC and 6.3 for McDonald's.

The second chain-specific variable is the number of new outlets opened in a city during a calendar year. The average number of new outlets is 0.4 for KFC (with a SD of 1.7) and 0.2 for McDonald's (with a SD of 1.1). A notable feature of this variable in the data is the high frequency of zeros. Another notable feature is that the SD is many times higher than the mean. Both these features will influence our choice of the econometric model in the first stage of estimation below.

The third chain-specific variable is the distance from own nearest outlet. It is our measure of distance from own network. It takes non-zero values only for the cities in which the chain has yet to enter. This variable measures the geographic distance (in kilometers) between the city in question and the nearest city with at least one own outlet. The average distance from network is 327 kilometers for KFC (with a SD of 442 kilometers) and 647 kilometers for McDonald's (with a SD of 1,095 kilometers).

The fourth chain-specific variable is the network density. We define network density to be the weighted number of own outlets within a 300-kilometer radius, where the weights vary linearly and inversely with the distance. Specifically, we define the network density of chain $i$ in city $c$ at time $t$ as

$$
w_{i c t}=\sum_{k \mid d_{c k} \leq 300} n_{i k t}\left(1-\frac{d_{c k}}{300}\right),
$$

where $n_{i k t}$ is the number of chain $i$ 's outlets in city $k$ and $d_{c k}$ is the geographic distance between cities $c$ and $k$. The sum is over all cities within a 300-kilometers radius of city $c 13$ The average value of network density variable is 10.8 for KFC (with a SD of 24.0) and 7.9 for McDonald's (with a SD of 18.6). This network density variable is similar to the one in Nishida (2013) except for two differences: 1) while we add the number of outlets, weighted by distance between the cities, he adds the number of markets; 2) while we count the number of own outlets within a 300-kilometer radius, he counts the number of adjacent markets with at least one own outlet.

The last two variables in Table 5 are city-specific. The first is population density (in units of thousands per square kilometer). The average population density is 1.21 with a

\footnotetext{
${ }^{13}$ We also constructed this variable with $100 \mathrm{~km}, 200 \mathrm{~km}, 400 \mathrm{~km}, 500 \mathrm{~km}$ and $600 \mathrm{~km}$ radii. If $w^{r}$ denotes the variable based on a radius of $r$ kilometers, the correlations between $w^{300}$ and $\left\{w^{100}, w^{200}, w^{400}, w^{500}, w^{600}\right\}$ are $\{0.98,0.99,0.98,0.94,0.90\}$ for KFC and $\{0.99,1.00,1.00,0.99,0.99\}$ for McDonald's. We use $w^{300}$ as our benchmark. The variants based on other radii give similar results.
} 
SD of 1.12. The second variable is the gross domestic product (GDP) per capita (in 2001 prices) in thousands of yuans. The average GDP per capita is $¥ 14.8$ thousand with a SD of ¥15.8 thousand.

In addition to the above, we have also collected data on city-level operating income. These data are available from 2003 to 2007 for an unbalanced panel of cities . We summarize these data in Table 6 and provide more details about them in Appendix $\mathrm{A}$. The average annual operating income per city for KFC is $¥ 328.57$ million (with a SD of $¥ 489.83$ million) and for McDonald’s it is ¥83.04 million (with a SD of ¥141.36 million). The average annual income per outlet is ¥17.91 million ( $\mathrm{SD}=¥ 20.29$ million) for KFC and $¥ 9.26$ million ( $\mathrm{SD}=¥ 5.44$ million) for McDonald’s.

\section{Model}

We have argued in Section 2.1 that KFC and McDonald's can reasonably be considered a duopoly of foreign fastfood chains in the Chinese market over the sample period. Hence we model their entry and expansion decisions as a dynamic duopoly game 14 Due to the data constraint, we assume that the decisions to open new outlets are made at the city level ${ }^{15}$ Instead, if we assumed that a chain chose the number and the locations of all its new outlets in the country simultaneously at the national level, the problem would be similar to those in Jia (2008) and Nishida (2013). The complexity of those problems is well known. Our assumption makes an extremely large problem very tractable ${ }^{16}$

The strategy of modeling the outlet opening decisions at the city level has its limitations. First, fastfood chains generally decide expansion policies at the national level and then look for suitable markets to implement the policies. We take care of this problem by introducing the age of a chain as a state variable. This is motivated by the observation that both KFC and McDonald's expanded very slowly during the first few years after their entry into the Chinese market. Later, after gaining some experience in the market, they expanded rapidly. Second, the decisions to open new outlets in a city are likely to be affected by own (and possibly rival's) presence in the neighboring cities. In other words, we cannot assume these markets to be isolated and independent of one another. We take care of this problem by constructing two separate state variables for each chain: 1) distance from the nearest

\footnotetext{
${ }^{14}$ Our model does not feature exit from a city or a reduction in the number of outlets within a city. This is because we do not observe exit or contraction in our sample. In our original dataset, we do not have complete information on $39 \mathrm{KFC}$ and 24 McDonald's outlets. Because these account for a small fraction of the total number of outlets in the data and also there is no particular pattern for the missing data, we exclude them from our sample. The outlets with missing information may include exits.

${ }^{15} \mathrm{We}$ have 4428 city-year observations in our dataset. If we were to model the outlet opening decisions at the national level, we would only have 18 yearly observations.

${ }^{16}$ The cardinality of the choice set for each chain in our model is just 51: a chain can open any number of outlets from 0 to 50 in a city in one year. If we modelled the same problem at the national level, with 246 markets in our sample, the cardinality of the choice set for each chain would be $51^{246}$.
} 
city with at least one own outlet; and 2) network density 17 The first variable is likely to affect the decision to enter a new city. A chain is more likely to enter a new city if it is closer to its existing network of outlets Holmes [2011)]. The second variable is likely to have implications for the expansion decision. A denser network may have both positive and negative effects on a chain's ability to expand further. An important simplifying assumption of our model is that these variables (distance and network density) evolve exogenously and we estimate their evolution from the data. When the chains make their entry and expansion decisions, they take the evolution of these variables as given. We further discuss and justify this assumption in Section 3.4 .

In our model, time is discrete and planning horizon is infinite. There are two fastfood chains, $i$ and $j 18$ Each period, for each of the $C$ cities, they simultaneously choose the number of outlets to open in the city. Although the chains make their decisions at the city level, their decisions depend on the networks of own and rival's outlets in nearby cities and also on their own expansion policy at the national level (see below). In the following, we model the problem of chain $i$. Chain $j$ solves a similar problem.

\subsection{Choice and State Variables}

The choice variable for chain $i$ is $x_{i} \in\{0,1, \ldots, \bar{x}\}$, where $\bar{x}$ is the maximum number of outlets that a chain can open in a city in one period. The length of a period is one year. The state variables are: 1) $n_{i c} \in\{0,1, \ldots, \bar{n}\}$, number of own outlets in city $c$ at the beginning of the period; 2) $n_{j c} \in\{0,1, \ldots, \bar{n}\}$, number of rival's outlets in the city at the beginning of the period; 3) $a_{i} \in\{0,1, \ldots, \bar{a}\}$, age of the chain in the country; 4) $d_{i c} \in D$, distance from the nearest city with at least one own outlet; 5) $d_{j c} \in D$, distance from the nearest city with at least one rival outlet; 6) $w_{i c} \in W$, own network density;7) $w_{j c} \in W$, rival's network density; 8) $\mathbf{z}_{c}$, a vector of city characteristics; 9) $\varepsilon_{i c}^{e}$, a privately observed random shock to the cost of entering a new market; and 10) $\varepsilon_{i c}^{s}$, a privately observed random shock to the sunk cost of opening a new outlet. For the ease of notation, we define the state vector $\mathbf{s}_{i c}=\left\{n_{i}, n_{j}, a_{i}, d_{i c}, d_{j c}, w_{i c}, w_{j c}, \mathbf{z}_{c}, \varepsilon_{i c}^{e}, \varepsilon_{i c}^{s}\right\}$.

We now provide some details on the state variables. We treat all outlets of a chain to be homogenous within a city in terms of profits. We allow the outlets of the two chains to be different from one another in terms of profit per customer, fixed cost of operations, etc. We model variables $d$ and $w$ as discrete variables with exogenously given transition matrices. We estimate the transition matrices, separately for each chain, from the data. Vector $\mathbf{z}_{c}$ contains city characteristics such as population, area, real GDP per capita, etc.

\footnotetext{
${ }^{17}$ We have defined these variables in Section 2.2

${ }^{18}$ If $i$ denotes KFC then $j$ denotes McDonald's and vice versa.
} 


\subsection{Timing of Events}

The timing of events for chain $i$ in city $c$ within a period is as follows. At the start of the period the chain observes its state vector $\mathbf{s}_{i c}$. Given $\mathbf{s}_{i c}$, and its beliefs about the strategy of its rival, the chain decides on $x_{i c}$, the number of new outlets to open in the city during the period. It then pays, if applicable, the cost of entry into the city, $c_{i}^{e}\left(\mathbf{s}_{i c}\right)$, and the sunk cost of opening new outlet(s) $c_{i}^{s}\left(\mathbf{s}_{i c}\right)$. Chain $j$ makes a similar decision simultaneously. All new outlets are opened for business at the end of the period and the state vector is updated to $\mathbf{s}_{i c}^{\prime}$. We discuss the state transitions in more detail in Section 3.5 .

\subsection{Static Profit Function}

Our primary interest is in modeling a chain's decisions about entry into and expansion within a city. Both entry and expansion are dynamic decisions and we describe them in some detail in the next subsection. Here we describe a chain's static profit function. A static profit function is appropriate for a fastfood firm because fastfood is neither a durable nor a storable good. We define profit for chain $i$ in city $c$ as: ${ }^{19}$

$$
\pi_{i c}=\underbrace{\left[(p p m)_{i} q_{i}\left(n_{i c}, n_{j c}, P_{c}\right)-F_{i}\right]}_{\text {Profit per outlet }} n_{i c} .
$$

The expression in the square brackets is profit per outlet. ppm is profit per meal. This is simply the difference between the price of a meal and the marginal cost of producing the meal. We assume all meals of a chain to be homogenous. $q$ is the number of meals per outlet per year. We assume $q$ to be a function of the number of own outlets in the city $\left(n_{i c}\right)$, the number of the rival's outlets in the city $\left(n_{j c}\right)$ and the city's population $\left(P_{c}\right) . F_{i}$ is the fixed cost of running an outlet. Following the literature on static games of entry [Bresnahan and Reiss (1991), Berry (1992), Toivanen and Waterson (2005), Jia (2008), etc.] we adopt a reduced-form approach and assume that $q_{i}$ is a linear function of $n_{i c}, n_{j c}$ and $P_{c}{ }^{20}$ This translates into the following reduced-form equation for profits: ${ }^{21}$

$$
\pi_{i c}\left(\mathbf{s}_{i c} \mid \boldsymbol{\theta}_{i}^{\pi}\right)=\left[\theta_{i 0}^{\pi}+\theta_{i n_{i}}^{\pi} n_{i c}+\theta_{i n_{j}}^{\pi} n_{j c}+\theta_{i P}^{\pi} P_{c}\right] n_{i c}
$$

\footnotetext{
${ }^{19}$ We suppress the time subscripts because the profit function is static. The profit function of chain $j$ is similarly defined.

${ }^{20} \mathrm{We}$ adopt a reduced-form approach because data on prices and market shares are not available. Our primary interest is in how entry and expansion decisions are influenced by the presence of the rival conditional on demand side being given.

${ }^{21}$ We denote most of the parameters in this paper by Greek letter $\theta$, and use superscripts and subscripts to distinguish among them. We use superscripts to identify whether a parameter belongs to the profit function (superscript $\pi$ ) or the cost of entry function (superscript $e$ ) or the sunk cost of opening a new outlet (superscript $s$ ). We use $i$ in the subscript to highlight that there are separate parameters for chains $i$ and $j$. The second symbol in the subscript, after $i$, identifies the variable to which that coefficient belongs. For example, for parameter $\theta_{i n_{i}}^{\pi}$ in (3), $n_{i}$ in the subscript tells us that this parameter measures the effect of the number of own outlets on profit per outlet.
} 
The parameter vector $\boldsymbol{\theta}_{i}^{\pi}$ consists of 4 parameters: $\left\{\theta_{i 0}^{\pi}, \theta_{i n_{i}}^{\pi}, \theta_{i n_{j}}^{\pi}, \theta_{i P}^{\pi}\right\}$. Note that we cannot separately identify fixed cost or the profit per meal. However, we do not need to do so because all we need is to define profit as a function of state ${ }^{22}$ We have chosen the above specification because it is simple, parsimonious and we can estimate it by ordinary least squares (OLS). We can also estimate the parameters in the profit function together with the structural parameters subject to a scale restriction. We shall say more about the estimation of the profit functions in Section 4.

To test the robustness of our structural parameter estimates we also try another specification for the profit function. To derive this specification, think about a city with population $P_{c}$. Assume that a fraction $f$ of population eats Western fastfood. Among those who eat the Western fastfood, each individual eats an average of $l$ meals per year. She divides these meals between the two chains in proportion to their number of outlets in the city. So she eats $l \times \frac{n_{i}}{n_{i}+n_{j}}$ meals at chain $i$ outlets and $l \times \frac{n_{j}}{n_{i}+n_{j}}$ meals at chain $j$ outlets ${ }^{23}$ Each meal generates a profit of $p_{i}$ yuans for chain $i$ and $p_{j}$ yuans for chain $j$. Finally, the fixed cost of operating an outlet is $\theta_{i f}^{\pi}$ for chain $i$ and $\theta_{j f}^{\pi}$ for chain $j$. Given these assumptions, the period profit of chain $i$ from city $c$ is given by

$$
\pi_{i c}\left(\mathbf{s}_{i c} \mid \boldsymbol{\theta}_{i}^{\pi}\right)=\left(\theta_{i P /\left(n_{i}+n_{j}\right)}^{\pi} \frac{P_{c}}{n_{i c}+n_{j c}}-\theta_{i f}^{\pi}\right) n_{i c}
$$

where $\theta_{i P /\left(n_{i}+n_{j}\right)}^{\pi}=p_{i} l f$. This specification is more restrictive than the one in $(3)$ as it imposes an arbitrary functional form on the variable profit. Once again, like the specification in (3), we can estimate this profit function either by OLS or by normalizing one of the parameters and estimating the rest together with the structural parameters.

\subsection{Dynamic Problem}

The dynamic problem of chain $i$ is to choose $x_{i c}$, the number of new outlets to open in the city during the period, to maximize the expected value of future profit streams. We can write this problem in the form of the following Bellman's equation:

$$
\begin{aligned}
V_{i}\left(\mathbf{s}_{i c} \mid \boldsymbol{\theta}_{i}, \chi_{j}\right)= & \max _{x_{i c}}\left\{\left[\pi_{i}\left(\mathbf{s}_{i c} \mid \boldsymbol{\theta}_{i}^{\pi}\right)+\mathbf{1}\left(n_{i c}=0\right) \min \left(x_{i c}, 1\right) c_{i}^{e}\left(\mathbf{s}_{i c} \mid \boldsymbol{\theta}_{i}^{e}\right)\right.\right. \\
& \left.\left.+c_{i}^{s}\left(\mathbf{s}_{i c}, x_{i c} \mid \boldsymbol{\theta}_{i}^{s}\right) x_{i c}\right]+\beta E V_{i}\left(\mathbf{s}_{i c}^{\prime} \mid \boldsymbol{\theta}_{i}, \chi_{j}\right)\right\} .
\end{aligned}
$$

Note that the value function has $i$ in the subscript. It implies that the two chains can potentially have different value functions. This is motivated by the reduced-form regression

\footnotetext{
${ }^{22}$ Other state variables that could potentially explain profit are GDP per capita and age of the chain. We did include them in some earlier specifications but their effect on profit was statistically insignificant.

${ }^{23}$ If chain $i$ is more popular than chain $j$ and gets a more than proportional share of customers, we can incorporate this by multiplying $n_{i}$ with a scalar greater than 1 . Another way to do so would be to assume that chain $i$ generates more profit per meal than does chain $j$. Our empirical strategy is closer to the latter assumption.
} 
results that we report later in Section 5.1. We take the reduced-form results as preliminary evidence that the profits and the costs of entry and expansion could potentially be asymmetric across chains and give rise to different value functions. For the same reason, functions $\pi_{i}(\cdot), c_{i}^{e}(\cdot)$ and $c_{i}^{s}(\cdot)$ are also subscripted by $i$.

The value function in (5) is defined at the city level. The spillovers from own and rival's outlets in neighboring cities are captured by the distance $\left(d_{i c}\right.$ and $\left.d_{j c}\right)$ and network $\left(w_{i c}\right.$ and $w_{j c}$ ) variables. The country-level expansion policy is captured by the age variable $a_{i}$. The age, distance and network variables also serve another important purpose in the model. In the dynamic problem in (5), the chain maximizes value at the city level and does not take into account the effect (positive or negative) of its decisions in the city on the value of the chain in other cities. Variables $a_{i}, d_{i c}$ and $w_{i c}$ capture the interdependence across cities indirectly. Although variables $d_{i c}$ and $w_{i c}$ evolve exogenously, because their evolution and the parameters associated with them are estimated from the data, they capture the collective effects of city-level decisions in a summary way.

We now define and explain various components of (5). The value function in (5) depends on state vector $\mathbf{s}_{i c}=\left\{n_{i}, n_{j}, a_{i}, d_{i c}, d_{j c}, w_{i c}, w_{j c}, \mathbf{z}_{c}, \varepsilon_{i c}^{e}, \varepsilon_{i c}^{s}\right\}$, conditional on $\boldsymbol{\theta}_{i}$ and $\chi_{j}$. The vector $\boldsymbol{\theta}_{i}=\left\{\beta, \boldsymbol{\theta}_{i}^{\pi}, \boldsymbol{\theta}_{i}^{e}, \boldsymbol{\theta}_{i}^{s}\right\}$ contains all the parameters of the model that we aim to estimate for chain $i$. We describe these parameters in some detail in Section 4.1. The function $\chi_{j}$ represents chain $i$ 's beliefs about the strategy profile of the rival chain $j$. We assume a rational expectation equilibrium and hence in equilibrium the expected strategy profile of the rival coincides with the equilibrium strategy profile ${ }^{24}$

The chain chooses $x_{i c}$ to maximize the expression inside the curly braces. The optimal solution to this problem is a strategy profile $\chi_{i}\left(\mathbf{s}_{i c} \mid \boldsymbol{\theta}_{i}, \chi_{j}\right)$. We say more about it when we define equilibrium in Section 3.6 .

The expression contained in square brackets in (5) represents the period return from the city. The first term in the square brackets, $\pi_{i}\left(\mathbf{s}_{i c} \mid \boldsymbol{\theta}_{i}^{\pi}\right)$, is the static profit function for chain $i$. We have already discussed the static profit function in Section 3.3 .

The second term in the square brackets is $\mathbf{1}\left(n_{i c}=0\right) \min \left(x_{i c}, 1\right) c_{i}^{e}\left(\mathbf{s}_{i c} \mid \boldsymbol{\theta}_{i}^{e}\right)$. It is related to the cost of entering a new city. The first component, $\mathbf{1}\left(n_{i c}=0\right)$, is an indicator variable that takes the value 1 if the chain does not have an outlet in the city at the beginning of the period, i.e. $n_{i c}=0$. So the cost of entry is only relevant if the chain is opening outlets in the city for the first time. The second component, $\min \left(x_{i c}, 1\right)$, implies that even if a chain opens more than one outlet during the period of entry, it pays the cost of entry only once 25 The third component, $c_{i}^{e}\left(\mathbf{s}_{i c} \mid \boldsymbol{\theta}_{i}^{e}\right)$, is the cost of entry, which is explicitly defined in equation (7) below. The parameters in vector $\boldsymbol{\theta}_{i}^{e}$ will inform us, among other things, about the effects of own and rival's networks of outlets on the cost of entry.

\footnotetext{
${ }^{24}$ Also see the definition of equilibrium in Section 3.6 below.

${ }^{25}$ In our dataset, in about $14 \%$ of the cases (32 cities out of 230 for KFC and 20 cities out of 142 for McDonald's) a chain opens more than one outlet during the calendar year of its entry into a new city.
} 
The third term in the square brackets is $c_{i}^{s}\left(\mathbf{s}_{i c}, x_{i c} \mid \boldsymbol{\theta}_{i}^{s}\right) x_{i c}$. It represents the sunk cost of opening $x_{i c}$ new outlets in the city. The first component, $c_{i}^{s}\left(\mathbf{s}_{i c}, x_{i c} \mid \boldsymbol{\theta}_{i}^{s}\right)$, is the sunk cost of opening a single outlet. The second component, $x_{i c}$, is the number of new outlets opened in the city during the period. We define $c_{i}^{s}(\cdot)$ explicitly in equation (9). The parameters in vector $\boldsymbol{\theta}_{i}^{s}$ will inform us about the effects of own and rival's networks on the sunk cost of opening a new outlet and hence on the expansion decisions.

The entry and sunk cost terms enter equation (5) with positive signs. This implies that if these costs are positive then we shall have $c_{i}^{e}\left(\mathbf{s}_{i c} \mid \boldsymbol{\theta}_{i}^{e}\right)<0$ and $c_{i}^{s}\left(\mathbf{s}_{i c}, x_{i c} \mid \boldsymbol{\theta}_{i}^{s}\right)<0$. The reason for writing them in this way is that we would like to have a negative sign for every parameter that contributes negatively to the period return function and a positive sign for every parameter that contributes positively. The benefit of doing so will be clear when we present and discuss the estimation results in Section 5.2 .

The last term in (5) is the present discounted continuation value. Parameter $\beta \in(0,1)$ is the discount factor. $E V_{i}$ is the expected continuation value, where the expectation is over the evolution of $\mathbf{s}_{i c}$ and over the probability distributions of the rival's private shocks.

\subsection{State Transitions}

We now turn to the evolution of state variables in the model. The evolution of $n_{i c}$ and $n_{j c}$, conditional on choice, is straight forward. Using a prime $\left(^{\prime}\right)$ to denote next period values

$$
\begin{aligned}
& n_{i c}^{\prime}=n_{i c}+x_{i c}, \text { and } \\
& E\left(n_{j c}^{\prime}\right)=n_{j c}+E\left(x_{j c}\right) .
\end{aligned}
$$

There is an expectation sign on $x_{j c}$ because chain $i$ does not observe the private cost shocks of its rival.

We assume that the state variables $\left\{d_{i c}, d_{j c}, w_{i c}, w_{j c}\right\}$ follow first-order Markov processes that we estimate from the data. By construction, variables $d_{i c}$ and $d_{j c}$ decline over time as the chains expand their network of outlets. Hence the transition probability matrices for these variables are lower triangular. The absorbing state for these variables is 0 , which is reached when a chain opens its first outlet in the city, i.e. when $n_{i c}>0, d_{i c}=0$. The same is true for chain $j$. Variables $w_{i c}$ and $w_{j c}$ are weakly increasing over time and hence have upper-triangular transition matrices. We assume these variables to enter an absorbing state when they take values greater than $100{ }^{26}$

The city characteristic vector $\mathbf{z}$ includes area, population and GDP per capita. We assume area to be constant over time. ${ }^{27}$ We assume population and GDP per capita to follow first-order Markov processes that we estimate from the data.

\footnotetext{
${ }^{26}$ In our dataset, the variable $w$ exceeds 100 in only $2.2 \%$ of the cases for KFC and $1.1 \%$ of the cases for McDonald's.

${ }^{27}$ The area of some cities in the sample is not constant due to changes in the administrative jurisdiction of the cities over time.
} 
Age, $a_{i}$, increases by one unit every period deterministically until it reaches the absorbing state $\bar{a}$. Once $a_{i}=\bar{a}$, it remains constant. We set $\bar{a}=50$. Here the implicit assumption is that the additional benefits from more experience dissipate after a chain has been in the country long enough.

The private shocks to the cost of entry $\left(\varepsilon_{i}^{e}\right)$ and sunk cost of opening a new outlet $\left(\varepsilon_{i}^{s}\right)$ are assumed i.i.d. over time, across cities and across the two chains. They are drawn from zero mean normal distributions with $\sigma_{i}^{e}$ and $\sigma_{i}^{s}$ standard deviations.

\subsection{Equilibrium}

We use the concept of Markov Perfect Equilibrium (MPE). The MPE of our model consists of strategy profiles $\chi_{i}^{*}\left(\mathbf{s}_{i c} \mid \boldsymbol{\theta}_{i}, \chi_{j}^{*}\right)$ and $\chi_{j}^{*}\left(\mathbf{s}_{j c} \mid \boldsymbol{\theta}_{j}, \chi_{i}^{*}\right)$ such that $\chi_{i}^{*}\left(\mathbf{s}_{i c} \mid \boldsymbol{\theta}_{i}, \chi_{j}^{*}\right)$ is an optimal solution to the dynamic program in (5) and $\chi_{j}^{*}\left(\mathbf{s}_{j c} \mid \boldsymbol{\theta}_{j}, \chi_{i}^{*}\right)$ is an optimal solution to a similar program for chain $j$.

\section{Estimation}

In this section we discuss issues related to the estimation of our model in some detail. In Section 4.1 we specify the functional forms and list the parameters to be estimated. In Section 4.2 we discuss our estimation methodology. In Section 4.3 we talk about identification and in Section 4.4 we define our measures of cost spillovers.

\subsection{Parameters to Estimate}

The objective of estimation is to estimate parameters in vector $\boldsymbol{\theta}_{i}=\left\{\boldsymbol{\theta}_{i}^{\pi}, \boldsymbol{\theta}_{i}^{e}, \boldsymbol{\theta}_{i}^{s}\right\}{ }^{28}$ We now describe the parameters in some detail.

The number of parameters in vector $\boldsymbol{\theta}_{i}^{\pi}$ depends on the specification of the profit function. If we adopt the specification in (3), then the vector $\boldsymbol{\theta}_{i}^{\pi}$ consists of the following four parameters: $\left\{\theta_{i 0}^{\pi}, \theta_{i n_{i}}^{\pi}, \theta_{i n_{j}}^{\pi}, \theta_{i P}^{\pi}\right\}$. Instead, if we adopt the specification in (4), the vector $\boldsymbol{\theta}_{i}^{\pi}$ consists of the following two parameters: $\left\{\theta_{i P /\left(n_{i}+n_{j}\right)}^{\pi}, \theta_{i f}^{\pi}\right\}$.

Parameters in vector $\boldsymbol{\theta}_{i}^{e}$ are related to the cost of entry into a city. We parameterize it as

$c_{i}^{e}\left(\mathbf{s}_{i c t} \mid \boldsymbol{\theta}_{i}^{e}\right)=\theta_{i 0}^{e}+\theta_{i P / A}^{e} P_{c t} / A_{c}+\theta_{i y}^{e} y_{c t}+\theta_{i a_{i}}^{e} a_{i t}+\theta_{i a_{i}^{2}}^{e} a_{i t}^{2}+\theta_{i d_{i}}^{e} d_{i c t}+\theta_{i d_{j}}^{e} d_{j c t}+\theta_{i n_{j}}^{e} n_{j c t}+\sigma_{i}^{e} \epsilon_{i c t}^{e}$.

Note that $n_{i}$ does not appear in (7) because the entry cost is paid only if $n_{i}=0$ (and $\left.x_{i} \geq 1\right)$. ${ }^{29}$ Parameter $\theta_{i 0}^{e}$ is the fixed cost of entry which is independent of other factors.

\footnotetext{
${ }^{28}$ We do not include the discount factor $\beta$ in the list of the parameters to be estimated. Our dataset does not allow us to identify the discount factor.

${ }^{29}$ We do not include density variables in the specification of entry costs because they are highly correlated
} 
Parameters $\theta_{i P / A}^{e}$ and $\theta_{i y}^{e}$ measure the effects of population density and GDP per capita on the cost of entry. Parameters $\theta_{i a_{i}}^{e}$ and $\theta_{i a_{i}^{2}}^{e}$ measure the effect of experience in the country on the cost of entry. As a chain becomes more experienced, it may enter new cities at a lower cost. Parameters $\theta_{i d_{i}}^{e}$ and $\theta_{i d_{j}}^{e}$ measure the effect of the proximity of own and rival's network on the cost of entry. If there are economies of density, one would expect a smaller distance from own or rival's network to contribute positively to the period return function and lower the cost of entry. Parameter $\theta_{i n_{j}}^{e}$ measures the effect of rival's presence in the city on the cost of entry: if there are positive information spillovers from prior presence of the rival in the city, we would expect a lower cost of entry. The last term in (7) is the private shock to the cost of entry. It has two components. The first component, $\epsilon_{i}^{e}$, is a standard normal variable and the second component, $\sigma_{i}^{e}$, which is another structural parameter to be estimated, is the standard deviation of the shock. The above parameterization of the cost of entry has a very simple interpretation. To see it clearly, we rewrite (7) as

$$
c_{i}^{e}\left(\mathbf{s}_{i c t} \mid \boldsymbol{\theta}_{i}^{e}\right)=\bar{c}_{i}^{e}\left(\mathbf{s}_{i c t} \mid \boldsymbol{\theta}_{i}^{e}\right)+\sigma_{i}^{e} \epsilon_{i c t}^{e} .
$$

Because we have assumed $\epsilon_{i}^{e}$ to be a standard normal variable, our parametrization of entry cost models it as a normally distributed variable with mean $\bar{c}_{i}^{e}$ and standard deviation $\sigma_{i}^{e}$.

Parameters in vector $\boldsymbol{\theta}_{i}^{s}$ are related to the sunk cost of opening a new outlet. We parameterize it a: 30

$$
\begin{aligned}
c_{i}^{s}\left(\mathbf{s}_{i c t} \mid \boldsymbol{\theta}_{i}^{s}\right)= & \theta_{i 0}^{s}+\theta_{i P / A}^{s} P_{c t} / A_{c}+\theta_{i y}^{s} y_{c t}+\theta_{i a_{i}}^{s} a_{i t}+\theta_{i a_{i}^{2}}^{s} a_{i t}^{2}+\theta_{i w_{i}}^{s} w_{i c t}+\theta_{i w_{j}}^{s} w_{j c t} \\
& +\theta_{i x_{i}}^{s} x_{i c t}+\theta_{i n_{i}}^{s} n_{i c t}+\theta_{i n_{j}}^{s} n_{j c t}+\sigma_{i}^{s} \epsilon_{i c t}^{s} .
\end{aligned}
$$

Parameter $\theta_{i 0}^{s}$ is the fixed cost of opening a new outlet. Parameters $\theta_{i P / A}^{s}$ and $\theta_{i y}^{s}$ measure the effects of population density and GDP per capita on the sunk cost of opening a new outlet. Parameters $\theta_{i a_{i}}^{s}$ and $\theta_{i a_{i}^{2}}^{s}$ measure the effect of experience on the sunk cost. Parameters $\theta_{i w_{i}}^{s}$ and $\theta_{i w_{j}}^{s}$ measure the effect of network density on the sunk cost. A denser own network may make it cheaper for the chain to open a new outlet. Parameter $\theta_{i x_{i}}^{s}$ measures the effect of the number of own new outlets being opened on the sunk cost. The idea is that the sunk cost per outlet may be different if more outlets are opened simultaneously. Parameters $\theta_{i n_{i}}^{s}$ and $\theta_{i n_{j}}^{s}$ measure the effect of own and rival's outlets in the city on the sunk cost of opening a new outlet. The private shock to the sunk cost of opening a new outlet is $\epsilon_{i}^{s} \sigma_{i}^{s}$, where $\epsilon_{i}^{s}$ is a standard normal variable and $\sigma_{i}^{s}$ is the standard deviation of the shock, which is another structural parameter to be estimated. This parameterization of sunk cost, just like that of entry cost above, has a simple interpretation. To see that, we

with the distance variables. The correlation between the distance and density variables is -0.31 for KFC and -0.24 for McDonald's. Both correlations are significant at $1 \%$ level.

${ }^{30}$ We do not include distance variable in $(9)$ because, by definition, the value of distance variable is zero after the chain has entered a city. 
rewrite $(9)$ as

$$
c_{i}^{s}\left(\mathbf{s}_{i c t} \mid \boldsymbol{\theta}_{i}^{s}\right)=\bar{c}_{i}^{s}\left(\mathbf{s}_{i c t} \mid \boldsymbol{\theta}_{i}^{s}\right)+\sigma_{i}^{s} \epsilon_{i c t}^{s} .
$$

Because we assume $\epsilon_{i}^{s}$ to be a standard normal variable, our estimated sunk cost will be normally distributed with mean $\bar{c}_{i}^{s}$ and standard deviation $\sigma_{i}^{s}$.

An implicit assumption behind the entry- and sunk-cost functions in (7) and (9) is that they depend on all relevant state variables. This may or may not be true. We include all relevant variables in our specifications and let the data decide which ones actually have a significant effect on these economic costs.

Given our research question about the spillovers from the rival and how they compare with the spillovers from own network of outlets, the parameters of interest in the entry-cost function are $\theta_{i d_{i}}^{e}, \theta_{i d_{j}}^{e}$ and $\theta_{i n_{j}}^{e}$. These parameters measure the effect on the cost of entry of own distance from the city, the rival's distance from the city and the number of rival's outlets in the city, respectively. The parameters of interest in the sunk-cost function are $\theta_{i w_{i}}^{s}, \theta_{i w_{j}}^{s}, \theta_{i n_{i}}^{s}$ and $\theta_{i n_{j}}^{s}$. The first two measure the effect of own and rival's network density on the sunk cost of opening a new outlet. The last two measure the effect of the number of own and rival's outlets in the city on the sunk cost. We define our measures of spillovers in Section 4.4

To sum up, in our benchmark specification there are 25 parameters to estimate for each chain: $\beta ; \boldsymbol{\theta}_{i}^{\pi}$ (4 parameters); $\boldsymbol{\theta}_{i}^{\boldsymbol{e}}$ (9 parameters) and $\boldsymbol{\theta}_{i}^{\boldsymbol{s}}$ (11 parameters). Of these, 24 are unique to each chain and $\beta$, the discount factor, is common across the chains. We cannot identify $\beta$ from our dataset so we assign it a fixed value and assume $\beta=0.90$. For the rest of the parameters, we discuss our estimation methodology in the following sub-section.

\subsection{Estimation Methodology}

Until recently the structural estimation of dynamic games was difficult due to the heavy computational burden of estimation and the problem of multiple equilibria. However, using the insight of Hotz and Miller (1993), a number of recent studies have proposed computationally efficient methods to estimate parameters of dynamic games. Leading papers in this literature include Aguirregabiria and Mira (2007), Bajari et al. (2007), Pakes et al. (2007) and Pesendorfer and Schmidt-Dengler (2008). To deal with the large state space in the dynamic game here, we employ the method proposed by Bajari et al. (2007), from here on BBL.

The estimation process has two stages. In the first stage, we estimate the static profit functions. We also estimate equilibrium policy functions under the assumption that the choices that we observe in the data represent a unique Markov Perfect Equilibrium of the game. In the same step, we also estimate the empirical transition probability matrices for the relevant variables. We then use the estimates of static profit functions, equilibrium policy functions and state transition matrices to compute numerical estimates of the value 
functions using forward simulations. We also compute the numerical estimates of the value function when the firm in question deviates from the observed equilibrium policy. Under the assumption of Markov Perfect Equilibrium, the values generated from equilibrium policies should be higher than those generated under alternative policies. If this is not the case, then our equilbirum condition is violated and we record the difference between the equilibrium and the alternative values. In the second stage, we pick the parameter vector that minimizes the squared sum of these differences over all alternative policies and all observed states of the world. We now explain the estimation steps in some detail.

\subsubsection{First Stage}

In the first stage we estimate the static profit functions, equilibrium policies, state-transition matrices and numerical value functions.

Static Profit Functions To estimate either of the two specifications of the static profit function in (3) and (4), we need city-level data on operating income, number of outlets (for both chains) and population. We have already described our dataset in Section 2.2. Our preferred estimation method for both specifications is OLS. We use (3) as our benchmark specification because it is simple to interpret and does not impose unnecessary restrictions on the profit function. We use (4) to test the robustness of our results. Although the parameters in the profit function can also be estimated together with the other structural parameters, such joint estimation runs into identification problems. We present the results of one such exercise in Section 6.3. There we also highlight the identification problem.

Equilibrium Policies A key element of BBL's methodology is the assumption that the data represent a unique Markov Perfect Equilibrium. Hence it is possible to estimate equilibrium policies directly from the data. Ideally this should be done non-parametrically but that would require a very large dataset. A more practical option is to use a flexible parametric specification.

We use Negative-Binomial (NB) models to estimate equilibrium policies from the data. The NB model is appropriate for our purpose because the dependent variable, i.e. the number of new outlets opened, is a count variable with many zero observations (see Table (4). Also, the variance of the number of new outlets far exceeds its mean (see Table 5), hence a NB model is more suitable than a simple Poisson model.

To estimate equilibrium policies from data, we regress the number of new outlets opened during a period on various state variables. We do so separately for the two chains. We comment on the estimated policies in Section 5.1 .

State-Transition Matrices In our model, there are six state variables that we assume to follow first-order Markov processes: GDP per capita; population; two distance variables 
$\left(d_{i}\right.$ and $\left.d_{j}\right)$ and two network variables $\left(w_{i}\right.$ and $\left.w_{j}\right)$. For all the six variables, we estimate the state-transition matrices from the data. In the following we explain how we do so using the distance variable $d_{i}$ as an example. We follow the same procedure for the other five variables. To estimate the state-transition matrices, we first discretize the variable ${ }^{31} \mathrm{We}$ make sure that the discretization is rich enough to capture important variation in the data. Let $p_{s, s^{\prime}}$ denote an element of the state-transition matrix, where $s$ is the current period state and $s^{\prime}$ is the next period state. To find the value of $p_{s, s^{\prime}}$, we count the observations in the data with the current state $s$ and the future state $s^{\prime}$. We then divide this number by the number of observations in the data with the current state $s$.

$$
p_{s, s^{\prime}}=\frac{\sum \mathbf{1}\left(\text { Current State }=s \text { and Next-period State }=s^{\prime}\right)}{\sum \mathbf{1}(\text { Current State }=s)},
$$

where $\mathbf{1}(\cdot)$ is an indicator function and the sums are over all observations in the sample.

Numerical Estimates of Value Function We now explain how to construct numerical estimates of the value functions using forward simulations. We omit city subscripts for ease of notation. Let $R_{i t}$ be the period return function and let $B_{i t}=\mathbf{1}\left(n_{i t}=0\right) \min \left(x_{i t}, 1\right)$. Using (5) we can write the period return function as

$$
R_{i t}\left(\mathbf{s}_{i t} \mid \boldsymbol{\theta}_{i}, \chi_{i}^{*}, \chi_{j}^{*}\right)=\pi_{i}\left(\mathbf{s}_{i t} \mid \boldsymbol{\theta}_{i}^{\pi}\right) n_{i t}-B_{i t} c_{i}^{e}\left(\mathbf{s}_{i t} \mid \boldsymbol{\theta}_{i}^{e}\right)-c_{i}^{s}\left(\mathbf{s}_{i t} \mid \boldsymbol{\theta}_{i}^{s}\right) x_{i, t} .
$$

Note that the period return function is based on both chains playing their equilibrium strategies $\chi_{i}^{*}$ and $\chi_{j}^{*}$. We start from an arbitrary initial state $\mathbf{s}_{i 0}$ and use the first-stage estimated policy functions to get $x_{i 0}$ and $x_{j 0}$. Using $\mathbf{s}_{i 0}, x_{i 0}$ and $x_{j 0}$, and a given parameter vector $\boldsymbol{\theta}_{i}$, we compute the numerical value of $R_{i 0}$. Using $x_{i 0}$ and $x_{j 0}$, and nature's draws for other state variables, we get $\mathbf{s}_{i 1}$ and then repeat the above steps to get $R_{i 1}$. Continuing this way, we get the sequence $\left\{R_{i t}\right\}_{t=0}^{T}$, where $T$ is an arbitrarily chosen large number such that $\beta^{T}$ is close to zero 32 The present discounted value of these return functions gives us one numerical estimate of the value function:

$$
V_{i}\left(\mathbf{s}_{i 0} \mid \boldsymbol{\theta}_{i}, \chi_{i}^{*}, \chi_{j}^{*}\right)=\sum_{t=0}^{\infty} \beta^{t} R_{i t} .
$$

We repeat this process a large number of times to reduce the simulation error due to the randomness of the Markov processes and the private cost shocks. The average over these simulations is our numerical estimate of the value function for a given state and a given set of policy functions. We also compute the numerical estimates of the value function under

\footnotetext{
${ }^{31}$ In Table C.1 we show how we discretize the distance variable.

${ }^{32}$ When we forward simulate, we impose an upper limit of 500 outlets in a city. This number is reasonable because in our dataset the maximum number of KFC outlets is 191 (in Shanghai in 2007) and McDonald's outlets is 117 (in Beijing in 2007). Even in the large US cities, where McDonald's and KFC outlets are ubiquitous, the total number of outlets of these chains seldom exceeds 500. For example, the total number of McDonald's outlets is 546 in New York city, 478 in Los Angeles and 398 in Chicago. The corresponding numbers for KFC are: 233, 221 and 154. [Source: yellowpages.com, last accessed on March 7, 2013]
} 
alternative policies. We randomly draw 100 alternative policy parameter vectors according to

$$
\theta^{p^{\prime}}=\theta^{p}(1+\varepsilon)
$$

where $\varepsilon \sim N(0,1), \theta^{p}$ is the equilibrium policy parameter vector and $\theta^{p^{\prime}}$ is the alternative policy parameter vector. Under both equilibrium and alternative policies, the number of outlets to open is bounded between 0 and $\bar{x}$, both inclusive. We set $\bar{x}=5033$ If $\chi_{i}^{l}$ denotes the $l$ th alternative policy, then $V_{i}\left(\mathbf{s}_{i 0} \mid \boldsymbol{\theta}_{i}, \chi_{i}^{l}, \chi_{j}^{*}\right)$ denotes the numerical estimate of the value function when chain $i$ follows policy $l$ and its rival chain $j$ follows the equilibrium policy.

\subsubsection{Second Stage}

If $\chi_{i}^{*}$ is indeed the Markov Perfect Equilibrium policy then the following must hold at the true parameter vector $\boldsymbol{\theta}_{i}^{*}$.

$$
V_{i}\left(\mathbf{s}_{i 0} \mid \boldsymbol{\theta}_{i}^{*}, \chi_{i}^{*}, \chi_{j}^{*}\right) \geq V_{i}\left(\mathbf{s}_{i 0} \mid \boldsymbol{\theta}_{i}^{*}, \chi_{i}^{l}, \chi_{j}^{*}\right) .
$$

But the inequality may not hold for some states if $\boldsymbol{\theta}_{i}^{*}$ is replaced by an alternative parameter vector $\boldsymbol{\theta}_{i}$. BBL propose to estimate $\boldsymbol{\theta}_{i}^{*}$ by minimizing the violations of the last inequality. More formally, let us define a difference variable $d$ as

$$
d\left(\mathbf{s}_{i 0} \mid \boldsymbol{\theta}_{i}^{*}, \chi_{i}^{l}, \chi_{j}^{*}\right)=V_{i}\left(\mathbf{s}_{i 0} \mid \boldsymbol{\theta}_{i}^{*}, \chi_{i}^{*}, \chi_{j}^{*}\right)-V_{i}\left(\mathbf{s}_{i 0} \mid \boldsymbol{\theta}_{i}^{*}, \chi_{i}^{l}, \chi_{j}^{*}\right)
$$

and a criterion function $Q$ as

$$
Q\left(\boldsymbol{\theta}_{i}\right)=\sum_{\mathbf{s}_{i 0}} \sum_{l=1}^{L}\left[\min \left(d\left(\mathbf{s}_{i, 0}, \mathbf{z}_{0} \mid \boldsymbol{\theta}_{i}, \chi_{i}^{l}, \chi_{j}^{*}\right), 0\right)\right]^{2},
$$

where the first sum is over all states in the state space and the second sum is over $L$ alternative policies ${ }^{34}$ The BBL point estimate of $\boldsymbol{\theta}_{i}^{*}$ is then given by

$$
\hat{\boldsymbol{\theta}}_{i}=\arg \min _{\boldsymbol{\theta}_{i}} Q\left(\boldsymbol{\theta}_{i}\right)
$$

\subsection{Identification}

In BBL's method, the parameters of a model are point identified if there is a unique parameter vector that satisfies all possible equilibrium inequalities implied by (15). In practice, to make estimation computationally feasible, one has to work with a sample analog of $Q\left(\boldsymbol{\theta}_{i}\right)$ and compute it using a small subset of all possible initial states and a limited set of alternative policies. Srisuma (2010) discusses problems associated with this choice. He shows that it is possible to lose a lot of identification information if the alternative policies are

\footnotetext{
${ }^{33}$ In our dataset the maximum number of outlets that KFC has ever opened in a city within a year is 33 (in Shanghai in 2004). The maximum for McDonald's is 27 (in Shanghai in 2006).

${ }^{34}$ In our application $L=100$.
} 
not carefully chosen. He suggests that the set of alternative policies should be determined by how the state variables are transformed into an action. In other words, the alternatives should be informative about the structural parameters.

In our application we base $Q\left(\boldsymbol{\theta}_{i}\right)$ on all 3,786 unique states that we observe in the data. For each state, we consider 100 alternative policies by randomly perturbing each parameter in the estimated policy function according to (14). This gives us 378,600 equilibrium conditions to estimate 20 dynamic parameters in our baseline specification for each chain. We show below that these random perturbations are rich enough to uncover the underlying structural parameters.

In our model, all the dynamic parameters that we estimate appear in one of the following two functions: $c_{i}^{e}\left(\mathbf{s}_{i t} \mid \boldsymbol{\theta}_{i}^{e}\right)$ and $c_{i}^{s}\left(\mathbf{s}_{i t} \mid \boldsymbol{\theta}_{i}^{s}\right)$. These functions are explicitly defined in (7) and (9). Some state variables appear in both these functions. How do we separately identify the structural parameters associated with these state variables? In the following, we answer this question by using the state variable GDP per capita $\left(y_{i}\right)$ as an example. The same reasoning can be applied to other state variables that appear in both (7) and (9).

Because the value function is linear in structural parameters (see (13)), we can explicitly write the components of $d\left(\mathbf{s}_{i 0} \mid \boldsymbol{\theta}_{i}^{*}, \chi_{i}^{l}, \chi_{j}^{*}\right)$ (see (16) ) that depend on the state variable $y_{i}$ as: 35

$$
d\left(\mathbf{s}_{i 0} \mid \boldsymbol{\theta}_{i}^{*}, \chi_{i}^{l}, \chi_{j}^{*}\right)=\theta_{i y_{i}}^{e} \underbrace{\left(\sum_{t=0}^{T} \beta^{t} y_{i t} B_{i t}^{*}-\sum_{t=0}^{T} \beta^{t} y_{i t} B_{i t}^{l}\right)}_{A_{1}\left(\mathbf{s}_{i 0} \mid \chi_{i}^{l}, \chi_{j}^{*}\right)}+\theta_{i y_{i}}^{s} \underbrace{\left(\sum_{t=0}^{T} \beta^{t} y_{i t} x_{i t}^{*}-\sum_{t=0}^{T} \beta^{t} y_{i t} x_{i t}^{l}\right)}_{A_{2}\left(\mathbf{s}_{i 0} \mid \chi_{i}^{l}, \chi_{j}^{*}\right)}
$$

where $\left\{B_{i t}^{*}, x_{i t}^{*}\right\}_{t=0}^{T}$ sequences are generated by equilibrium policy $\chi_{i}^{*}$ and $\left\{B_{i t}^{l}, x_{i t}^{l}\right\}_{t=0}^{T}$ sequences are generated by alternative policy $\chi_{i}^{l}$. As long as $A_{1}\left(\mathbf{s}_{i 0} \mid \chi_{i}^{l}, \chi_{j}^{*}\right)$ and $A_{2}\left(\mathbf{s}_{i 0} \mid \chi_{i}^{l}, \chi_{j}^{*}\right)$ are not perfectly correlated across different initial states and different alternative policies, we should be able to separately identify the two parameters $\left(\theta_{i y_{i}}^{e}\right.$ and $\left.\theta_{i y_{i}}^{s}\right)$. Our simulations suggest that, in general, such correlations are very small.

At a more intuitive level, parameters in the entry cost function are identified from the variation in entry time due to a particular state. For example, if a chain enters a high GDP per capita city earlier than a low GDP per capita city, other things being equal, this would imply that a higher level of GDP per capita in a city lowers the cost of entry. Similarly the parameters in the sunk cost function are identified from the variation in the number of outlets opened due to a particular state. For example, if chain $i$ opens more outlets in cities with a higher number of own existing outlets, other things being equal, it would imply that more own outlets reduce the sunk cost of opening new outlets in the city.

\footnotetext{
${ }^{35}$ Each $d(\cdot)$ is based on a single initial state $\mathbf{s}_{i 0}$ and a single alternative policy $\chi_{i}^{l}$. In our application, there are 378,600 such $d$ 's that enter the criterion function $Q(\cdot)$.
} 


\subsection{Measurement of Spillovers}

In our model, the rival can affect a chain's cost of entry in one of the two ways.

1. If the rival is not present in the city in which the chain is considering entry then the distance from the nearest city in which the rival is present $\left(d_{j}\right)$ may affect the cost of entry. This effect is measured by parameter $\theta_{i d_{j}}^{e}$.

2. If the rival is already present in the city, the extent of the rival's presence, measured by the rival's number of outlets $n_{j}$, may affect the cost of entry. This effect is measured by parameter $\theta_{i n_{j}}^{e}$.

Both these variables, $d_{j}$ and $n_{j}$, affect $\bar{c}_{i}^{e}$, the mean of the distribution of entry cost (see (8)). Our measure of spillovers due to a certain variable is the parameter corresponding to that variable expressed in units of standard deviations. For example, we measure the entry-cost spillovers due to $d_{j}$ by $\theta_{i d_{j}}^{e} / \sigma_{i}^{e}$. The interpretation of this measure of spillovers is simple: it measures the change in the average cost of entry caused by a unit change in $d_{j}$ in units of standard deviations. Likewise, we measure the entry-cost spillovers due to $n_{j}$ by $\theta_{i n_{j}}^{e} / \sigma_{i}^{e}$.

We apply the same idea to measure spillovers from own network of outlets. For example, we measure the entry-cost spillovers due to the presence of own outlets in the nearby cities by $\theta_{i d_{i}}^{e} / \sigma_{i}^{e}$.

Once a chain has entered a city, there are two ways in which the rival can affect the chain's sunk cost of opening a new outlet.

1. The density of the rival's outlets in and around the city, measured by $w_{j}$, may affect the sunk cost of the chain. This effect is measured by parameter $\theta_{i w_{j}}^{s}$.

2. The number of rival's outlets in the city, which we denote by $n_{j}$, may also affect the sunk cost. This effect is measured by parameter $\theta_{i n_{j}}^{s}$.

The two variables, $w_{j}$ and $n_{j}$, affect $\bar{c}_{i}^{s}$, the mean of the distribution of the sunk cost (see (10)). Once again, we measure the spillovers by the size of the relevant parameter in units of standard deviations. Specifically, we measure the sunk-cost spillovers due to the rival's network density by $\theta_{i w_{j}}^{s} / \sigma_{i}^{s}$, and those due to the rival's number of outlets by $\theta_{i n_{j}}^{s} / \sigma_{i}^{s}$.

Similarly we measure sunk-cost spillovers due to own network density by $\theta_{i w_{i}}^{s} / \sigma_{i}^{s}$ and those due to own number of outlets by $\theta_{i n_{i}}^{s} / \sigma_{i}^{s}$.

\section{Estimation Results}

Estimation proceeds in two stages as we explained in Section 4. In the following we discuss the estimation results for each stage separately. 


\subsection{First-Stage Estimates}

In the first stage we estimate the static profit functions, equilibrium policies, state-transition matrices and numerical value functions. In the following, we discuss each of these estimates in some detail.

\subsubsection{Static Profit Functions}

The estimates of the static profit functions are in Table 7. The estimates in the upper panel are based on the specification of profit function in (3) and those in the lower panel are based on the specification in (4).

The estimates for KFC in the upper panel suggest that it earns an average annual profit of about ¥17.234 million from an outlet. This profit declines by ¥0.129 million with every additional own outlet (the cannibalization effect). Each additional rival outlet depresses the profit by about ¥0.022 million (the competition effect). Our reduced-form estimates of cannibalization and competition effects are qualitatively similar to the structural estimates in Aguirregabiria and Magesan (2012) except that for McDonald's they estimate the cannibalization effect to be positive, though statistically insignificant, in 2 of their 3 specifications (see their Table 10). We estimate that the overall population of the city has a positive effect on profits per outlet: an increase of a million people in population, boosts the profit per outlet by about $¥ 1$ million ${ }^{36}$ The estimates for McDonald’s in the upper panel suggest an average annual profit of $¥ 10.228$ million per outlet. An additional own outlet reduces the profit by ¥0.022 million and an additional rival’s outlet reduces it by ¥0.005 million. The effect of population is estimated to be negative, though it is statistically insignificant.

The estimates in the lower panel suggest positive fixed costs and a positive effect of population per outlet on profits. These parameters are hard to interpret due to a very restrictive specification of the profit function. We only use these estimates to test the robustness of our structural parameter estimates.

Because these static profits are the gains that the chains expect to reap forever when they enter a new market or expand within an existing market, they will affect our estimates of dynamic parameters in a non-trivial way. One can simply think of these profit functions as normalizations that help us estimate dynamic parameters. A doubling of all the parameters in the profit functions will lead to a doubling of all dynamic parameters. Hence, our measures of spillovers that we introduced in Section 4.4 are independent of such rescaling.

If the static profit functions are so fundamental to our dynamic parameter estimates and we have limited data to precisely estimate them, then does it not put the entire estimation exercise into question? The answer is, no. We do not view our profit and cost estimates to be definitive. We view them as instructive about the trends in the data. We use them in a fully-specified model of entry and expansion to inform us about the underlying structural

\footnotetext{
${ }^{36}$ The population data used in estimation are in units of thousands.
} 
parameters. In this sense, this paper also has the flavour of an applied theory work. If more detailed data on operating income become available, one can re-estimate our model to get more precise parameter estimates ${ }^{37}$ We also perform comprehensive robustness checks in Section 6.3 to show that the main conclusions of this study are robust to various parameterizations of the profit function.

\subsubsection{Equilibrium Policies}

Our equilibrium policy estimates are in Table 8. The dependent variable is the number of new outlets that a chain opens in a city within a year. Explanatory variables include a full set of state variables and, in some cases, their higher order and interaction terms as well. The state variables may affect the entry and expansion decisions differently. To account for this we define a "potential entrant" indicator variable, $I_{i}$, which takes the value 1 if chain $i$ has not yet entered the city, and 0 otherwise. To capture the differential effects of the state variables on entry and expansion, we interact the potential entry indicator variable with the state variables. For example consider the effect of GDP per capita on KFC's entry and expansion decisions. If KFC has yet to enter a city, the effect is positive and the parameter estimate is $0.024(=0.008+0.016)$. If KFC is already in the city, the effect is still positive but the parameter estimate is just 0.008 .

We estimate separate policy functions for the two chains. Our results justify this choice and we find that the two chains repond quite differently to some state variables. We estimate highly significant over-dispersion parameter for both chains. This justifies our choice of a Negative Binomial model over a Poisson model. The value of $R^{2}$ is 0.306 for KFC and 0.289 for McDonald's. These suggests that our regressions can explain close to one-third of the variation in the number of new outlets.

\subsubsection{State Transition Matrices}

We estimate the state transition matrices using the procedure that we described in Section 4.2.1. We report the estimated matrices in Appendix C (Tables C.2 to C.7). The transition matrices for GDP per capita and population are the same for the two chains. The transition matrix for GDP per capita, Table C.2, shows that although it is a persistent variable, it generally has a high probability (around a quarter) of going up. This reflects the rapid growth of the Chinese economy during the sample period (1990-2007). The transition matrix for population shows a much higher level of persistence and some probability (mostly around $4 \%$ ) of increase. This increase is mainly due to urbanization.

\footnotetext{
${ }^{37}$ We also tried to structurally estimate the profit functions together with the entry and sunk cost functions. To do so we normalized parameter $\theta_{i 0}^{\pi}$ to the abserved average profits per outlet. We present and discuss one such set of estimates in Section 6.3. where we also discuss the possible failure of identification for that estimation exercise.
} 
We estimate separate state-transition matrices for the distance variable for the two chains. The results are in Tables C.4 and C.5. The estimates show that when high, distance is more persistent for KFC. For example the probability of the distance staying within 2,000$3,000 \mathrm{~km}$ range is 0.84 for KFC and 0.62 for McDonald's. However, when low, distance is more persistent for McDonald's. For example, the probability of the distance staying in $50-100 \mathrm{~km}$ range is 0.89 for McDonald's and 0.80 for KFC.

We report the state-transition matrices for network density in Tables C.6 and C.7. It takes some time to build the network so at the lower levels, network density is more persistent for both chains. However, once the chains get going, the growth is quite rapid: the pobabilities of going up are very high for the 2-5 range. Although the growth appears to slow down at higher values, it is primarily because of the wider range for the individual bins that we have chosen while discretizing this variable.

\subsection{Second-Stage Estimates}

The structural parameter estimates are in Table 9. All the estimates are in millions of yuans. As we discussed in Section 4.3, there are 378,600 equilibrium conditions in our estimation algorithm. At the bottom of the table we report the percentage of the equilibrium conditions that are satisfied at the optimum.

We report the standard errors in the parentheses under the relevant coefficient estimates. These are bootstrapped standard errors. We randomly choose 30 subsamples of 123 cities each, with replacement, from a total of 246 cities. For each subsample we re-estimate the equilibrium policies, state transition matrices and the structural parameters. Let $\hat{\boldsymbol{\theta}}_{i}$ denote the vector of estimated structural parameters based on the full sample and $\hat{\boldsymbol{\theta}}_{i m}$ denote the vector of the estimated structural parameters from the $m$ th subsample $(m=\{1,2, \ldots, 30\})$, then the estimated asymptotic covariance matrix of $\hat{\boldsymbol{\theta}}_{i}$ is given by

$$
\operatorname{Var}\left(\hat{\boldsymbol{\theta}}_{i}\right)=\frac{1}{30} \sum_{m=1}^{30}\left[\hat{\boldsymbol{\theta}}_{i m}-\hat{\boldsymbol{\theta}}_{i}\right]\left[\hat{\boldsymbol{\theta}}_{i m}-\hat{\boldsymbol{\theta}}_{i}\right]^{\prime} .
$$

Although the computational burden of BBL's estimation method is low, it is nontrivial nonetheless. For example, the forward simulations to estimate the benchmark structural parameters for this paper took 24 days of CPU time and those to estimate the structural parameters from the 30 subsamples took a total of 375 days of CPU time 38

The estimates in Table 9 are our benchmark estimates. In the next section we use these estimates to answer the research question that motivated this paper: are there cost spillovers from the rival's network of outlets and how do they compare with the cost spillovers from own network?

\footnotetext{
${ }^{38}$ These simulations were run on HP Xeon Quad-core processors with CPU speeds of $1.7 \mathrm{GHz}$ each.
} 


\section{Spillovers}

We do three things in this section and devote a subsection to each of them. In Section 6.1, we explain and interpret the structural parameter estimates. In Section 6.2, we discuss the cost spillovers from the rival and compare them with the spillovers from own outlets. Finally, in Section 6.3, we perform some sensitivity checks on our structural parameter estimates.

\subsection{Interpretation of Estimates}

For the structural parameter estimates that we report in Table 9, a negative coefficient represents a negative contribution to the period return and hence an increase in cost. As stated earlier, all estimates are in millions of yuans. We start with entry cost parameter estimates that we report in the upper panel of Table 9. The estimates of $\theta_{i P / A}^{e}$ imply that an increase in population density of one thousand people per square kilometer reduces the cost of entry by ¥346.15 million for KFC and by ¥18.11 million for McDonald’s. The estimates of $\theta_{i y}^{e}$ imply that an increase in GDP per capita of one thousand yuans decreases the cost of entry by $¥ 21.57$ million for KFC and ¥1.07 million for McDonald’s. The estimates of $\theta_{i a}^{e}$ and $\theta_{i a^{2}}^{e}$ imply that the marginal effect of an increase of one year in national experience, proxied by age, is to reduce the cost of entry by $¥(76.23+91.92 a)$ million for KFC ${ }^{39}$ The marginal effect for McDonald’s is to change the cost of entry by $¥(11.21-0.98 a)$ million. Hence, for the first 11 years, age actually increased the cost of entry for McDonald's and it reduced it only by a small amount afterwards. The increase in the cost of entry with more national experience for McDonald's could be due to its capacity constraints in the national expansion process.

More relevant for our purpose are the estimates of $\theta_{i d_{i}}^{e}, \theta_{i d_{j}}^{e}$ and $\theta_{i n_{j}}^{e}$. The estimates of $\theta_{i d_{i}}^{e}$ suggest that if the city in question is one kilometer closer to a city with at least one own outlet, the cost of entry is lower by $¥ 3.01$ million for KFC and $¥ 0.21$ million for McDonald’s. In Figure B.1 we show, for all 216 cities that KFC entered from 1995 onwards, boxplots of distance from the nearest city with at least one own outlet. We do so for the year of entry as well as for the 5 consecutive years before entry. The median of the distribution drops from $238 \mathrm{~km}$ to just $105 \mathrm{~km}$ in five years. This decrease of $133 \mathrm{~km}$ in distance reduces the cost of entry by $¥ 400$ million for KFC. This effect is large and comparable to the effect on entry cost for KFC if the population density of the city went up by 1 standard deviation, say from 1.21 thousand people per square kilometer to 2.33. These estimates suggest the presence of the economies of density just as in Holmes (2011) and Aguirregabiria and Magesan (2012). The estimates of $\theta_{i d_{j}}^{e}$ suggest that if the city in question is one kilometer closer to a city with at least one rival outlet, the cost of entry is lower by ¥1.80 million for KFC and ¥0.62

\footnotetext{
${ }^{39}$ The contribution of age to the cost of entry of KFC is to reduce it by $76.23 a+45.96 a^{2}$. We differentiate it with respect to $a$ to get the marginal effect.
} 
million for McDonald's. So the cost of entry is influenced more by own distance than by rival's distance for KFC. For McDonald's, the rival's distance has a stronger effect. The estimates for $\theta_{i n_{j}}^{e}$ imply that if the rival has one more outlet in the city, the cost of entry is ¥220.18 million higher for KFC and ¥24.24 million lower for McDonald’s.

The overall story that our estimates tell about the chains' entry into new cities is the following. As KFC gains experience in the country, it expands rapidly focusing on more densely populated and richer cities first. It is also more likely to enter a city if the city is closer (as measured by distance) to its own network or to its rival's network. However, other things being equal, it is less likely to enter a city in which McDonald's is already present. In our dataset, KFC is the first mover in about $75 \%$ of the cities. The estimation results suggest that KFC is likely to make an initial entry into a comparable new market before entering McDonald's territories. The story for McDonald's is slightly different. In the first few years of operations in China, it grows cautiously and mostly follows KFC. It is more likely to enter the cities in which KFC is already present or that are closer to KFC's network.

We next comment on the sunk-cost parameter estimates that we report in the lower panel of Table 9. Population density and GDP per capita lower the sunk cost for both chains but their effect on the sunk cost is very small compared to that on the entry cost. The age effect on sunk cost of expansion is not significant for KFC. Age increases sunk cost for McDonald's but at a decreasing rate, and the effect is much smaller compared to the effect of age on the entry cost. An increase in own network density increases the sunk cost for KFC but decreases it for McDonald's. This may suggest that KFC is pursuing a "balanced" expansion strategy: it is more likely to expand in a city where the regional network density is relatively low than to add more outlets to a city where the regional network density is already high. McDonald's, on the other hand, is likely to open more outlets in a city with higher own network density. An increase in the rival's network density has the opposite effects for both chains. While KFC is more likely to expand in a city surrounded by more rival's outlets, McDonald's is less likely to add additional outlets when the rival's network is strong. The estimates of $\theta_{i x_{i}}^{s}$ imply that when a chain opens more outlets simultaneously, for example 2 instead of 1 , the sunk cost per outlet is higher. The estimates of $\theta_{i n_{i}}^{s}$ and $\theta_{i n_{j}}^{s}$ are positive and significant, implying that more own or rival outlets reduce the sunk cost, but again the magnitudes are small.

The overall story of expansion within a city is less interesting because the estimated parameters are small in value. Both chains expand more rapidly if there is a greater number of own or rival's outlets in the city. However, simultaneously opening more own outlets increases sunk cost. KFC expands faster in cities with higher per capita income. 


\subsection{Extent of Spillovers}

We compute the spillovers by using the methodology that we described in Section 4.4. The results are in Table 10. We begin our discussion of the results with the entry-cost spillovers from the rival's presence in the nearby cities. A 100-kilometer decrease in rival's distance decreases the entry cost by 1.22 SD's for KFC and by 1.52 SD's for McDonald's. These spillovers are substantial and they may be capturing multiple factors. For example, the rival's presence in the nearby cities may drive the local suppliers of fast-food chains to grow, which can benefit the entry of the focal chain. In addition, the entry of the rival in the region reduces the uncertainty and potentially saves the cost of exploring the market. How do these spillovers compare with spillovers from own presence in nearby cities? A 100-kilometer decrease in own distance decreases the entry cost by 2.03 SD's for KFC and by 0.51 SD's for McDonald's.

The second effect of a rival on entry cost comes from the rival's presence within the city. Our estimates suggest that a one-unit increase in rival's outlets increases entry cost by 1.49 SD's for KFC but decreases it by 0.59 SD's for McDonald's. The increase in KFC's entry cost could be the result of a combination of the following two factors: 1) it avoids the cities in which McDonald's is already present; 2) because it is the first mover in most of the cases, we estimate its implicit cost of entry to be lower for the cities where none of the two chains is present.

At this point, it is instructive to discuss the potential implications of these spillovers. To save on space, we focus on the effect of KFC's presence on McDonald's cost of entry. The implications of other spillovers can be analyzed in a similar way. Our estimates suggest that a one-unit increase in KFC's outlets decreases entry cost by 0.59 SD's for McDonald's. We arrived at this number by computing $\theta_{n_{j}}^{e} / \sigma_{i}^{e}(24.24 / 40.74)$. To see the implications of this number for McDonald's entry decisions, let us assume that the mean of McDonald's estimated entry-cost distribution is zero. Also assume that KFC's has $n_{j}$ outlets in the city. We can then plot the estimated distribution of McDonald's entry cost as a normal distribution with zero mean and 40.74 standard deviation. We plot this distribution in Figure 2 and mark it as $c_{i}^{e}\left(n_{j}\right)$. Now consider an increase in the number of KFC's outlets from $n_{j}$ to $n_{j}+1$. Assume that everything else remains the same. Because we estimate $\theta_{n_{j}}^{e}$ to be -24.24 , the estimated distribution of McDonald's entry cost will shift to the left by 24.24 units. The new distribution will have a mean of -24.24 but the same standard deviation. We also plot the new distribution in Figure 2 and mark it as $c_{i}^{e}\left(n_{j}+1\right)$. Given these distributions, we can compare the probability of McDonald's entry into the city when KFC's has $n_{j}$ outlets with the probability of entry when KFC's has $n_{j}+1$ outlets. We compute these probabilities for a range of expected benefits of entry and plot them in Panel (a) of Figure $3^{40}$ The curve marked $p_{i}^{e}\left(n_{j}\right)$ maps McDonald's expected benefit of entry into

\footnotetext{
${ }^{40}$ The expected benefit of entering a new city with one outlet is given by $\beta\left[E V_{i}\left(\mathbf{s}_{i c}^{\prime}, n_{i}=1 \mid \boldsymbol{\theta}_{i}, \chi_{j}\right)-\right.$ $\left.E V_{i}\left(\mathbf{s}_{i c}^{\prime}, n_{i}=0 \mid \boldsymbol{\theta}_{i}, \chi_{j}\right)\right]$. This benefit should be compared with the sum of entry and sunk costs. The
} 
its probability of entering the city when the number of KFC's outlets in the city is $n_{j}$. The curve marked $p_{i}^{e}\left(n_{j}+1\right)$ maps McDonald's expected benefit of entry into its probability of entering the city when the number of KFC's outlets in the city is $n_{j}+1$. When the expected benefit of entry is very low, say less than -150 , the probability of McDonald's entry is almost zero regardless of whether $\mathrm{KFC}$ has $n_{j}$ or $n_{j}+1$ outlets in the city. When the expected benefit is very high, say more than 100, the probability of McDonald's entry is almost 1 regardless of whether $\mathrm{KFC}$ has $n_{j}$ or $n_{j}+1$ outlets in the city. However, when the expected benefit of entry is between -150 and 100, the two probabilities are different. We plot their difference in Panel $(b)$ of Figure 3. For example, when the expected benefit of entry is zero and KFC has $n_{j}$ outlets in the city, the probability of McDonald's entry is slightly less than 0.5. However, if KFC has one more outlet in the city, the probability of McDonald's entry increases to slightly more than 0.7 . In other words, when the expected benefit of entry is 0 , one more KFC outlet increases McDonald's probability of entry by more than 0.2. This simple example illustrates that a spillover of 0.59 SD's can have substantial effects on the probability of entry.

To sum up, the spillovers from the rival are substantial in affecting the entry cost for both KFC and McDonald's. For McDonald's, both the rival's distance from, and its presence in, the city reduce the cost of entry. For KFC, the rival's distance reduces the cost but its presence in the city increases the cost.

Once a chain has entered the city, the spillovers from the rival in our model come from two sources: density of the rival's network in and around the city and the number of rival's outlets in the city. We report these spillovers in the lower panel of Table 10, A 10-unit increase in the rival's network density decreases the sunk cost by 0.03 SD's for KFC but increases it by 0.03 SD's for McDonald's. These effects are small. When we consider the effects of own network density, the magnitudes are slightly bigger but the signs are opposite for both chains. In summary, KFC is more likely to expand when the rival has a higher network density and when its own network density is lower. On the other hand, McDonald's is more likely to expand when the rival has a lower network density and its own network density is higher.

What about the effect of the number of rival's existing outlets on a chain's expansion? The estimates at the bottom of Table 10 suggest that these effects are negligible for both chains. Not only this, the effects from own existing outlets are also small for both chains. Hence, our estimates suggest that the effects of own or rival's existing outlets on expansion of a chain are very small. In Figure 4 we compare the effects of one more KFC outlet on McDonald's probabilities of entry and expansion. The expression $p_{i}^{e}\left(n_{j}+1\right)-p_{i}^{e}\left(n_{j}\right)$ denotes the difference in the probabilities of entry and it is exactly the same as the difference plotted in Panel $(b)$ of Figure 3 . Notice the difference in the units on $y$-axis between Figures

expected benefit, the entry cost and the sunk cost are all affected by the number of rival's outlets. However, to focus on the cost of entry, we assume that a change in the number of the rival's outlets only affects the cost of entry and does not affect the expected benefit or the sunk cost. 
3(b) and 4. This difference peaks at around 0.23 (Figure 3). It means that if KFC has one more outlet in the city, the probability of McDonald's entry could increase by up to 0.23 . The expression $p_{i}^{\exp }\left(n_{j}+1\right)-p_{i}^{\exp }\left(n_{j}\right)$ in Figure 4 denotes the difference in the probabilities of McDonald's expansion by one outlet if KFC has one more outlet in the city. This difference is almost negligible and even at its maximum it is less than 0.001. It means that if KFC has one more outlet in the city, it has hardly any effect on McDonald's probability of expansion.

We would like to make two comments about the results above. First, the estimates that we report are based on implicit entry and sunk costs that rationalize the observed choices as equilibirum outcomes. So when we say that a particular variable has very little effect on the sunk cost it implies that the observed decisions of the chain suggest so, under the assumption that the chain behaves according to our model. Second, the estimates and the effects that they imply are what we get from the data. We do not have a deep theory to explain why the estimates take the signs that they do and why their magnitude is what it is. So, for example, when we estimate an effect that is different between the two chains, we do not have a theory to explain why it is different.

\subsection{Robustness of the Results}

In this section we examine the sensitivity of our results to a number of variations on the benchmark model. To save on space, we focus on KFC. We report the first set of results in Table C.8. The results in column (1) are the benchmark estimates. They are the same as those reported in column (1) of Table 9 . Corresponding to the 7 columns in Table C.8, we report the 7 sets of spillover estimates in Table C.9.

Changing the Distribution of Cost Shocks For the benchmark estimates, we assumed that the shocks to entry and sunk costs follow a standard normal distribution. We change this assumption for the estimates in column (2) of Table C.8 and assume that the shocks to entry and sunk costs follow an extreme value distribution. The estimates barely change.

Estimating the Profit Function in the Second Stage For the estimates in column (3) of Table C.8, we fix $\theta_{i 0}^{\pi}$ and estimate the remaining parameters of the profit function together with the dynamic structural parameters. In one sense the joint estimation of profit and sunk-cost parameters is better than our benchmark approach of estimating the profit function parameters in the first stage and the sunk-cost parameters in the second stage. However, this is not our preferred approach because it is difficult to separately identify the parameters of the profit function from those of the sunk-cost function. To illustrate the failure of identification, we use the example of the state variable $n_{j}$, the number of rival's

outlet in the city. First we write the components of $d\left(\mathbf{s}_{i 0} \mid \boldsymbol{\theta}_{i}^{*}, \chi_{i}^{l}, \chi_{j}^{*}\right)$ (see 16]) that depend 
on the state variable $n_{j}$ as ${ }^{41}$

$$
\begin{aligned}
d\left(\mathbf{s}_{i 0} \mid \boldsymbol{\theta}_{i}^{*}, \chi_{i}^{l}, \chi_{j}^{*}\right)= & \theta_{i n_{j}} \underbrace{\left(\sum_{t=0}^{T} \beta^{t} n_{i t}^{*} n_{j t}^{*}-\sum_{t=0}^{T} \beta^{t} n_{i t}^{l} n_{j t}^{*}\right)}_{B_{1}\left(\mathbf{s}_{i 0} \mid \chi_{i}^{l}, \chi_{j}^{*}\right)}+\theta_{i n_{j}}^{e} \underbrace{\left(\sum_{t=0}^{T} \beta^{t} B_{i t}^{*} n_{j t}^{*}-\sum_{t=0}^{T} \beta^{t} B_{i t}^{l} n_{j t}^{*}\right)}_{B_{2}\left(\mathbf{s}_{i 0} \mid \chi_{i}^{l}, \chi_{j}^{*}\right)} \\
& +\theta_{i n_{j}}^{s} \underbrace{\left(\sum_{t=0}^{T} \beta^{t} x_{i t}^{*} n_{j t}^{*}-\sum_{t=0}^{T} \beta^{t} x_{i t}^{l} n_{j t}^{*}\right)}_{B_{3}\left(\mathbf{s}_{i 0} \mid \chi_{i}^{l}, \chi_{j}^{*}\right)}
\end{aligned}
$$

Recall from Section 4.3 that each $d(\cdot)$ is based on a single initial state $\mathbf{s}_{i 0}$ and a single alternative policy $\chi_{i}^{l}$. In order to separately identify $\theta_{i n_{j}}^{\pi}$ and $\theta_{i n_{j}}^{s}$, we need some independent variation between $B_{1}$ and $B_{3}$. Based on our forward simulation results the correlation between $B_{1}$ and $B_{3}$ is 0.987 for both KFC and McDonald's. Such high correlation renders separate identification of $\theta_{i n_{j}}^{\pi}$ and $\theta_{i n_{j}}^{s}$ difficult. On a related note, the correlation betwen $B_{1}$ and $B_{2}$ is mere 0.018 for KFC and 0.054 for McDonald's. This means that if we fix $\theta_{i n_{j}}^{s}$, we can separately identify $\theta_{i n_{j}}^{\pi}$ and $\theta_{i n_{j}}^{e}$. Likewise, the correlation betwen $B_{2}$ and $B_{3}$ is mere 0.016 for KFC and 0.047 for McDonald's. This means that if we fix $\theta_{i n_{j}}^{\pi}$, or estimate it separately as we do for our preferred specification, we can separately identify $\theta_{i n_{j}}^{e}$ and $\theta_{i n_{j}}^{s}$.

Regardless of whether we estimate the profit function parameters in the first stage or in the second stage, our conclusion about the spillovers remains the same: the spillovers to the entry cost are large and those to the sunk cost are neglibile. We can see this is column (3) of Table C.9.

Using a Different Profit Function For the estimates in column (4) of Table C.8, we use the static profit function in column (1) of the lower panel of Table 7. This specification of profit is more restrictive than our benchmark specification. The entry-cost spillovers in column (4) of Table C.9 are smaller than those in column (1), the benchmark case, but still quite substantial compared to the sunk-cost spillovers, which remain negligible.

Excluding Age Variable from the Model The age variable plays a very important role in our model. It proxies for the overall expansion policy of the chains in the country. For the estimates in column (5), we exclude the age variable from our model. The spillovers are smaller in this case (Table C.9 column (5)) but the same conclusion holds that the spillovers to the cost of entry are substantial and those to the sunk cost are negligible. An important difference from the benchmark spillovers is that in column (5) an increase in rival's number of outlets decreases the entry cost. Intuitively, when we exclude the age variable, the estimation routine wrongly assigns the positive effect from age to the rival's number of outlets. Also, when we exclude the age variable from the model, the percentage of equilibrium conditions that are satisfied at the final parameter estimates drops from $95.06 \%$

\footnotetext{
${ }^{41}$ In our application, there are 378,600 such $d$ 's that enter the criterion function $Q(\cdot)$.
} 
to $91.32 \%$, suggesting that the specification without the age variable is less satisfactory. We interpret these result as a validation of our decision to include age as a separate state variable in the model.

Understanding the Spillover Parameters One interpretation of our benchmark sunk-cost spillover parameters (Table C.8, column (1)) is that because we estimate negative cannibalization and compeition effects in the first stage $\left(\theta_{i n_{i}}^{\pi}=-0.129\right.$ and $\left.\theta_{i n_{j}}^{\pi}=-0.022\right)$, to rationalize the observed outcomes our structural model generates positive spillover parameters $\left(\theta_{i n_{i}}^{s}=1.60\right.$ and $\left.\theta_{i n_{j}}^{s}=0.20\right)$. We see a similar pattern in column (3) when we jointly estimate profit and sunk-cost parameters: $\theta_{i n_{i}}^{\pi}=-0.013$ and $\theta_{i n_{j}}^{\pi}=-0.002$ are both negative and $\theta_{i n_{i}}^{s}=0.28$ and $\theta_{i n_{j}}^{s}=0.03$ are both positive. What if we force $\theta_{i n_{i}}^{s}$ and $\theta_{i n_{j}}^{s}$ to be equal to zero and then estimate $\theta_{i n_{i}}^{\pi}$ and $\theta_{i n_{j}}^{\pi}$ using the structural model? The estimates of $\theta_{i n_{i}}^{\pi}$ and $\theta_{i n_{j}}^{\pi}$ would then show the net spillovers from own and rival outlets. We report the results of this experiment in column (6) of Table C.8. The estimates suggest that the net own spillovers are positive $\left(\theta_{i n_{i}}^{\pi}=0.011\right)$ and statistically significant. The spillovers from the rival are also positive $\left(\theta_{i n_{j}}^{\pi}=0.003\right)$ but not statistically significant. In column (7) we do the reverse: we set $\theta_{i n_{i}}^{\pi}$ and $\theta_{i n_{j}}^{\pi}$ equal to zero and use the structural model to estimate $\theta_{i n_{i}}^{s}$ and $\theta_{i n_{j}}^{s}$. The results suggest positive and statistically significant spillovers from both own and rival's outlets. Although these spillovers are very small when expressed in units of standard deviations, the results in columns (6) and (7) suggest that they are there and our structural model is able to identify them.

Changing the Parameters of the Profit Function In this set of robustness checks we change the parameter values in the static profit function, one at a time, to see how such specific changes affect our estimates of structural parameters and consequently the spillovers. The benchmark reduced-form profit function has four parameters: $\theta_{i 0}^{\pi}, \theta_{i n_{i}}^{\pi}, \theta_{i n_{j}}^{\pi}$ and $\theta_{i P}^{\pi}$. In Table C.10 we report structural estimates when each of these parameters is either set equal to zero or is doubled in value. The benchmark results are in column (1). In columns (8) and (9) we vary $\theta_{i 0}^{\pi}$, in columns (10) and (11) we vary $\theta_{i n_{i}}^{\pi}$, in columns (12) and (13) we vary $\theta_{i n_{j}}^{\pi}$ and in coulmns (14) and (15) we vary $\theta_{i P}^{\pi}$. Once again, corresponding to each column in Table C.10, we report spillovers in the corresponding column in Table C.11. In the following discussion, we focus on the spillovers and refer to the structural estimates only when needed.

In column (8) we set $\theta_{i 0}^{\pi}$ equal to zero. This change slightly reduces the size of entry-cost spillovers. The sunk-cost spillovers are more or less unchanged. In column (9), we double $\theta_{i 0}^{\pi}$ relative to its benchmark value. The entry-cost spillovers increase and the sunk-cost spillovers are again broadly the same.

In column (10), we set $\theta_{i n_{i}}^{\pi}$ equal to zero. This eliminates the demand cannibalization effect. The result is lower estimated spillovers. The intuition is that if more own outlets do not exert a negative effect on profit per outlet, we need smaller spillovers to rationalize the observed entry and expansion outcomes. In column (11), we set $\theta_{i n_{i}}^{\pi}$ equal to the double of 
its benchmark value. Although this change increases the cost estimates (see column (11) in Table C.10) across the board, it increases the estimated standard deviations of the cost shocks more than proportionally. Hence the spillovers, in units of SD's, are smaller.

In column (12), we set $\theta_{i n_{j}}^{\pi}$ equal to zero. The effect on spillovers is small because the benchmark estimate for this parameter was already very small. In column (13), when we double the size of this parameter, the spillovers increase. The effect on sunk-cost spillovers is much bigger than that on entry-cost spillovers. Nonetheless, the sunk-cost spillovers remain much smaller than the entry-cost spillovers.

In column (14), we set $\theta_{i P}^{\pi}$ equal to zero. This leads to smaller spillovers. In column (15) when we double $\theta_{i P}^{\pi}$ the entry-cost spillovers are larger but the sunk-cost spillovers are smaller. The sunk-cost spillovers are smaller because we estimate a much larger standard deviation of shocks to the sunk cost.

A general look at Table C.11 suggests that although there are important changes in the size of spillovers when we change the values of individual parameters in the static profit function, the spillovers to entry cost remain substantial and those to sunk cost remain relatively very small. We conclude from this robustness analysis that our broad finding of large entry-cost spillovers and small sunk-cost spillovers is robust to all the variations that we have tried in this sub-section. It is important to point out that the effects of various variations that we have tried are significant on the size of spillovers. It is just that the broad conclusions are not affected by them.

\section{Concluding Remarks}

We model the entry and expansion of KFC and McDonald's in the Chinese fastfood market as a dynamic game. We assume that the observed entry and expansion decisions are equilibrium outcomes. This allows us to recover the structural parameters of the game without solving for equilibrium. We use the estimated model to study the cost spillovers from the rival.

Our estimates suggest substantial spillovers to the cost of entering a new city. For example, if the rival is not present in the city in which the chain is entering and distance from the nearest city where the rival is present decreases by 100 kilometers, the cost of entry into the city decreases by 1.22 standard deviations for KFC and 1.52 standard deviations for McDonald's. If the rival is already present in the city, a one unit increase in the number of rival's outlets decreases the cost of entry by 0.59 standard deviations for McDonald's but increases it by 1.49 standard deviations for KFC.

We also find that the spillovers to the sunk cost of opening a new outlet are much smaller. For example, a 10-unit increase in the rival's network density decreases the sunk cost by 0.03 standard deviations for KFC and increases it by 0.03 standard deviations for McDonald's. Similary, the number of rival's outlets in the city also has a small effect on the sunk cost: a 
one unit increase in the rival's number of outlets decreases the sunk cost by approximately 0.01 standard deviations for both KFC and McDonald's. Hence the expansion within a city is not as much influenced by the presence of the rival as is the entry into a new city.

The very large state space of our dynamic model is a mixed blessing. On one hand, a rich set of state variables allows us to satisfy most of the equilibrium conditions during structural estimation and also provides better understanding of the determinants of entry and sunk costs. On the other hand, it makes the computation of equilibrium practically impossible and hence we cannot do any counterfactual analysis. An interesting counterfactual could have been to look at the entry and expansion of one of the two chains in the absence of the other.

We pick the structural parameters that rationalize the data in the light of our model. If the fastfood chains act according to a model that is radically different from ours, our structural parameter estimates may not reflect the true entry or sunk costs faced by the chains. This is not a limitation of our paper alone, this is a limitation of structural estimation in general: the structural estimates are as credible as the theory on which they are based.

We estimate the static profit functions using data from a small subset of cities. Future work should expand this dataset to make the static profit function estimates more credible. Also, we restrict our attention to the cost-side spillovers because of the lack of data on the demand side. Future work should extend this research to include estimation of the demand-side spillovers. 


\section{References}

Ackerberg, D., Benkard, C. L., Berry, S. \& Pakes, A. (2007), Econometric Tools for Analyzing Market Outcomes, in J. J. Heckman \& E. E. Leamer, eds, 'Handbook of Econometrics', Vol. 6A, Elsevier Science, Amesterdam: North-Holland.

Aguirregabiria, V. \& Magesan, A. (2012), Identification and Estimation of Dynamic Games when Players' Beliefs are not in Equilibrium. Working Paper 449, University of Toronto.

Aguirregabiria, V. \& Mira, P. (2007), 'Sequential Estimation of Dynamic Discrete Games', Econometrica 75(1), 1-53.

Bajari, P., Benkard, C. L. \& Levin, J. (2007), 'Estimating Dynamic Models of Imperfect Competition', Econometrica 75(5), 1331-1370.

Berry, S. \& Reiss, P. (2007), Empirical Models of Entry and Market Structure, in M. Armstrong \& R. H. Porter, eds, 'Handbook of Industrial Organization', Vol. 3, Elsevier Science, Amesterdam: North-Holland.

Berry, S. T. (1992), 'Estimation of a Model of Entry in the Airline Industry', Econometrica 60(4), 889-917.

Bresnahan, T. F. \& Reiss, P. C. (1991), 'Entry and Competition in Concentrated Markets', Journal of Political Economy 99(5), 977-1009.

Holmes, T. J. (2011), 'The Diffusion of Walmart and Economies of Density', Econometrica 79(1), 253-302.

Hotz, V. J. \& Miller, R. A. (1993), 'Conditional Choice Probabilities and Estimation of Dynamic Models', Review of Economic Studies 60(3), 497-529.

Jia, P. L. (2008), 'What Happens When Wal-Mart Comes to Town: An Empirical Analysis of the Discount Retailing Industry', Econometrica 76(6), 1263-1316.

Mazzeo, M. (2002), 'Product Choice and Oligopoly Market Structure', RAND Journal of Economics 33(2), 221-242.

Nishida, M. (2013), Estimating a Model of Strategic Network Choice: The ConvenienceStore Industry in Okinawa. Working Paper.

Pakes, A., Ostrovsky, M. \& Berry, S. (2007), 'Simple Estimators for the Parameters of Discrete Dynamic Games (with entry/exit examples)', Rand Journal of Economics 38(2), 373-399.

Pesendorfer, M. \& Schmidt-Dengler, P. (2008), 'Asymptotic Least Squares Estimators for Dynamic Games', Review of Economic Studies 75(3), 901-928. 
Rust, J. (1987), 'Optimal Replacement of GMC Bus Engines: An Empirical Model of Harold Zurcher', Econometrica 55(5), 999-1033.

Seim, K. (2006), 'An Empirical Model of Firm Entry with Endogenous Product-Type Choices', RAND Journal of Economics 37(3), 619-642.

Shen, Q. \& Xiao, P. (2013), McDonald's and KFC in China: Competitors or Companions? Forthcoming in Marketing Science.

Srisuma, S. (2010), Estimation of Structural Optimization Models: A Note on Identification. Working Paper, available at https://sites.google.com/site.tangsrisuma (last accessed on July 16, 2013).

Toivanen, O. \& Waterson, M. (2005), 'Market Structure and Entry: Where's the Beef?', The RAND Journal of Economics 36(3), 680-699.

Toivanen, O. \& Waterson, M. (2011), Retail Chain Expansion: The Early Years of McDonalds in Great Britain. Centre for Economic Policy Research, Discussion Paper No. 8534 .

Yang, N. (2012), An Empirical Model of Industry Dynamics with Common Uncertainty and Learning from the Actions of Competitors. Mimeo.

Zhu, T., Singh, V. \& Manuszak, M. D. (2009), 'Market Structure and Competition in the Retail Discount Industry', Journal of Marketing Research 46(4), 453-466. 
(a) Total Outlets

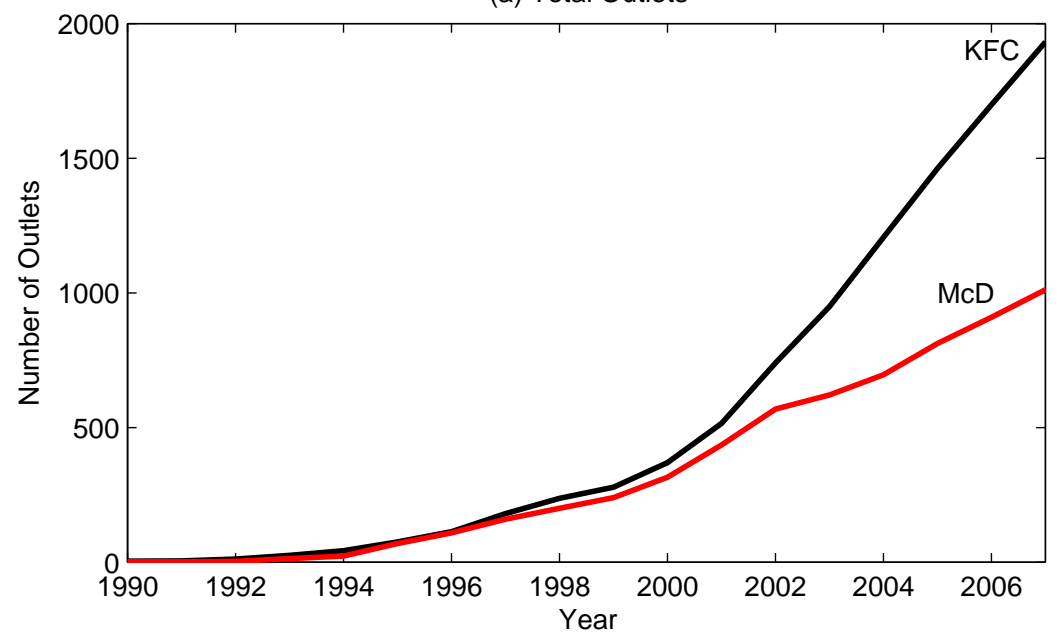

(b) Growth in Total Outlets

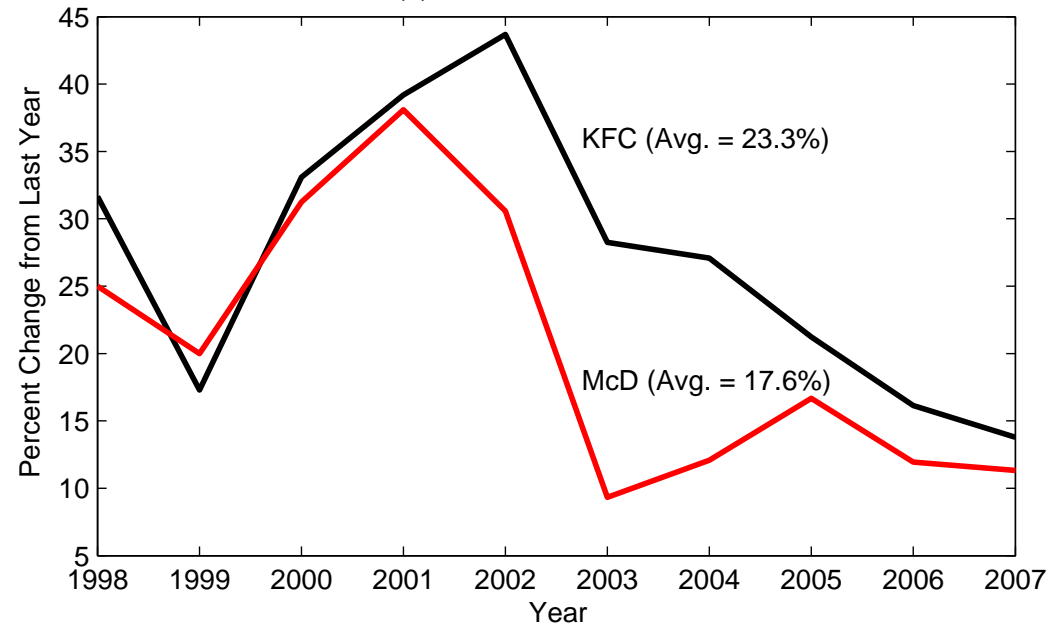

Figure 1: Total outlets in China (by the end of the year) and their growth 


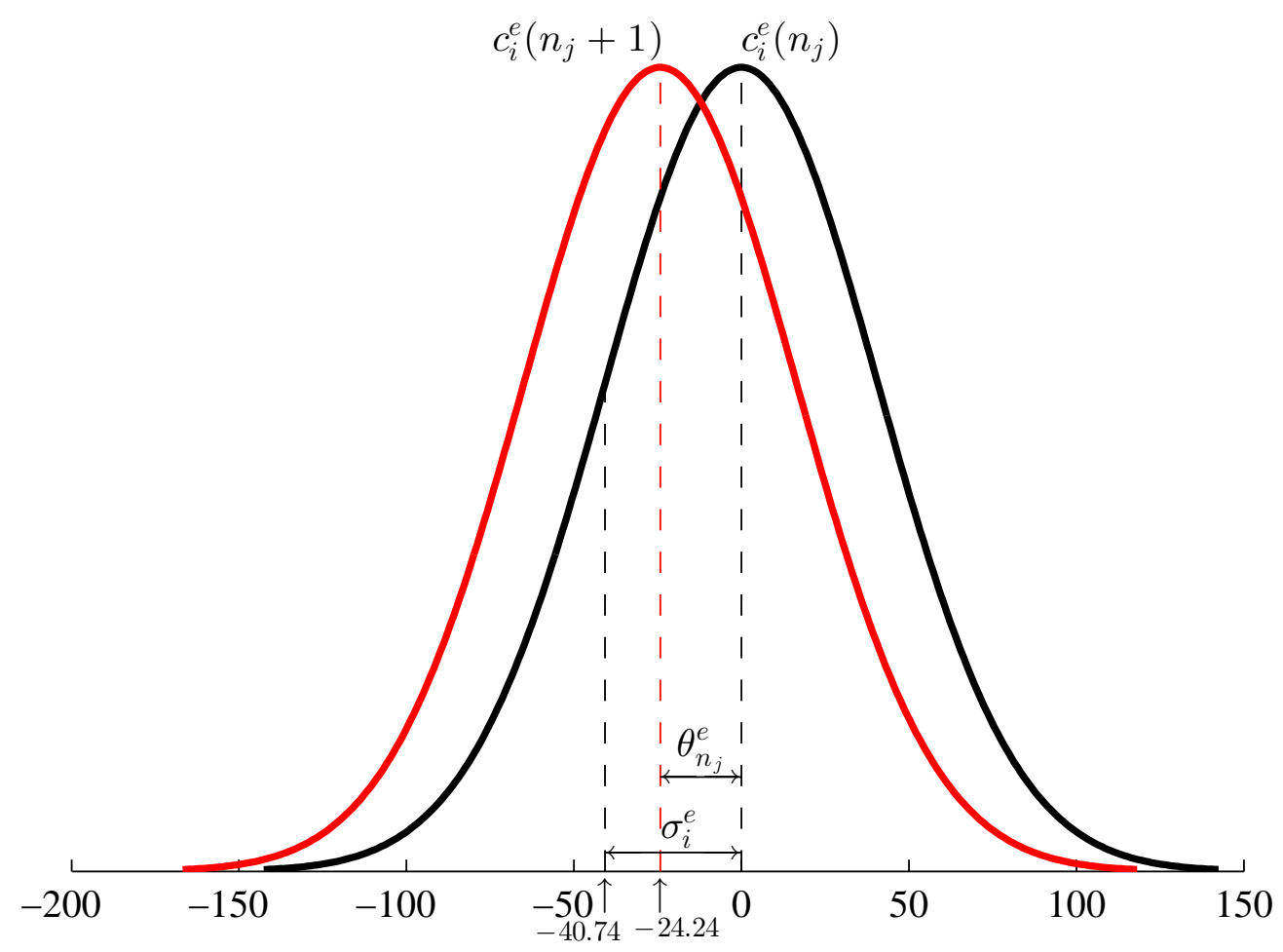

Figure 2: Shift in the distribution of McDonald's cost of entry when one more KFC outlet is present in the city 
(a)

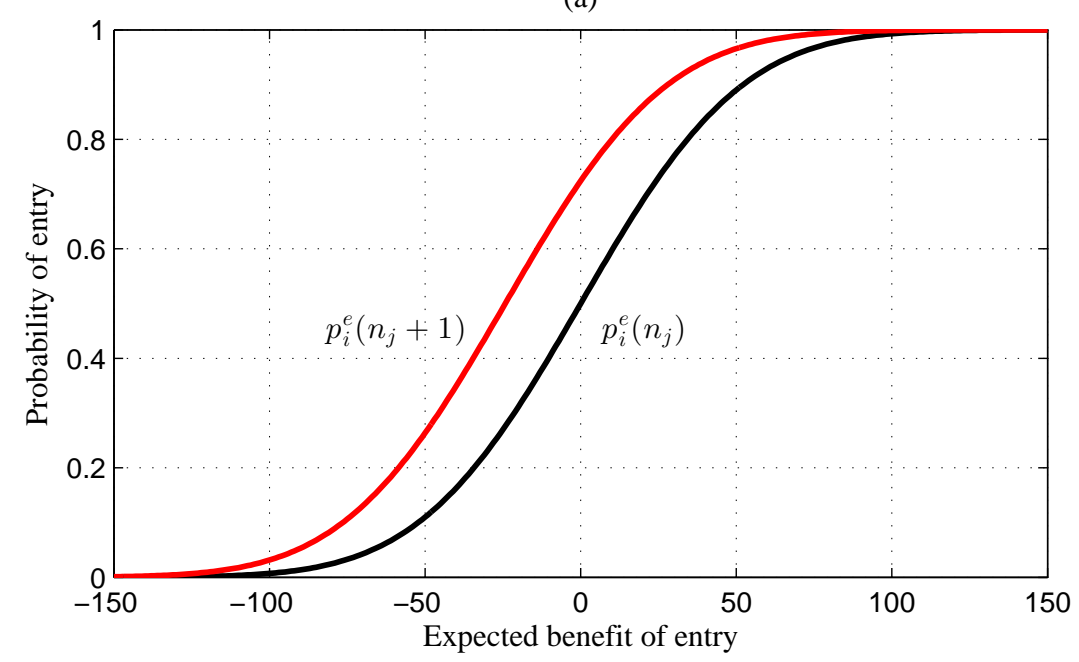

(b)

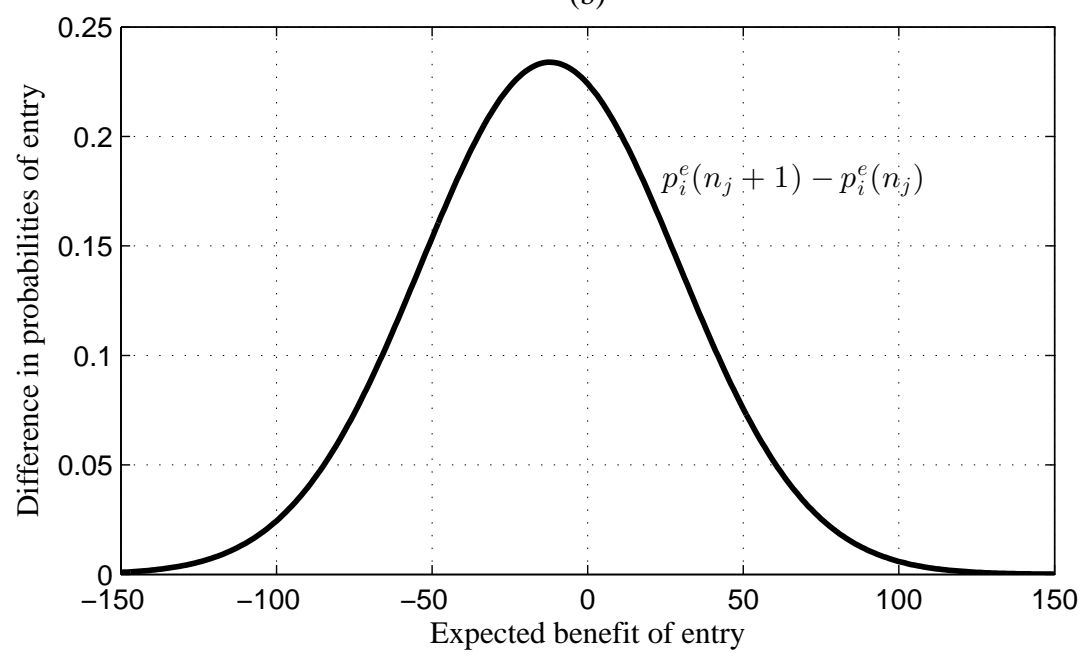

Figure 3: Changes in probabilities of McDonald's entry when one more KFC outlet is present in the city 


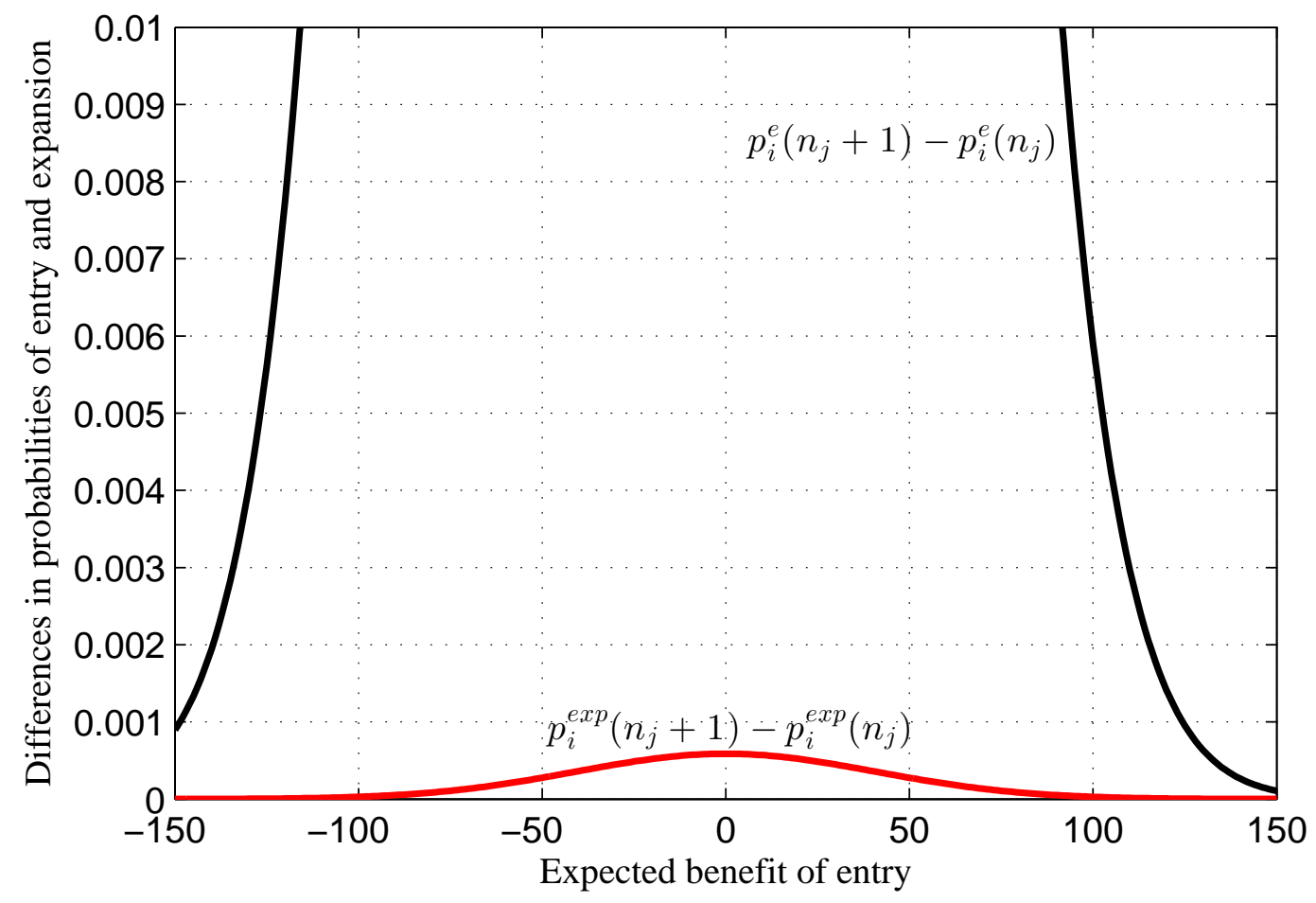

Figure 4: A Comparison of the effects of one more KFC outlet on McDonald's probabilities of entry and expansion 
Table 1: Number of cities entered and outlets opened (by the end of the year)

\begin{tabular}{cccccccc}
\hline \hline & \multicolumn{3}{c}{ KFC } & & \multicolumn{3}{c}{ McDonald's } \\
\cline { 2 - 4 } \cline { 6 - 8 } Year & Cities & Outlets & Outlets/City & & Cities & Outlets & Outlets/City \\
\hline 1990 & 2 & 4 & 2.0 & & 1 & 1 & 1.0 \\
1995 & 21 & 75 & 3.6 & & 11 & 69 & 6.3 \\
2000 & 72 & 370 & 5.1 & & 56 & 315 & 5.6 \\
2005 & 198 & 1462 & 7.4 & & 117 & 812 & 6.9 \\
2007 & 230 & 1932 & 8.4 & & 142 & 1012 & 7.1 \\
\hline \hline
\end{tabular}

Table 2: First mover

\begin{tabular}{lcc}
\hline \hline First Mover & No. of Cities & $(\%)$ \\
\hline KFC & 177 & $(75.0)$ \\
McDonald's & 33 & $(14.0)$ \\
Both & 26 & $(11.0)$ \\
\hline Total & 236 & $(100.0)$ \\
\hline \hline
\end{tabular}


Table 3: Frequency distribution of the number of outlets in a city (end of 2007)

\begin{tabular}{lccccc}
\hline \hline \multirow{2}{*}{$\begin{array}{l}\text { No. of } \\
\text { Outlets }\end{array}$} & \multicolumn{2}{c}{ KFC } & & \multicolumn{2}{c}{ McDonald's } \\
\cline { 2 - 3 } \cline { 5 - 6 } & No. of Cities & $(\%)$ & & No. of Cities & $(\%)$ \\
\hline 0 & 16 & $(6.5)$ & & 104 & $(42.3)$ \\
1 & 85 & $(34.6)$ & & 64 & $(26.0)$ \\
2 & 46 & $(18.7)$ & & 25 & $(10.2)$ \\
3 to 5 & 38 & $(15.4)$ & & 21 & $(8.5)$ \\
6 to 10 & 22 & $(8.9)$ & & 9 & $(3.7)$ \\
11 to 20 & 19 & $(7.7)$ & & 13 & $(5.3)$ \\
21 to 100 & 18 & $(7.3)$ & & 9 & $(3.7)$ \\
$>100$ & 2 & $(0.8)$ & & 1 & $(0.4)$ \\
\hline Total & 246 & $(99.9)^{\S}$ & 246 & $(100.1)^{\S}$ \\
\hline \hline
\end{tabular}

$\S_{\text {The sum does not add up to } 100 \text { due to rounding. }}$

Table 4: Frequency distributions of total and new outlets (Full sample: 246 cities, 18 years)

\begin{tabular}{ccccccccccc}
\hline \hline $\begin{array}{l}\text { Number of Total Outlets } \\
\text { (at the start of the year) }\end{array}$ & 0 & 1 & 2 & $3-5$ & $6-10$ & $11-20$ & $21-100$ & $>100$ & Total \\
\hline KFC: Frequency & 3,168 & 535 & 220 & 207 & 134 & 94 & 61 & 9 & 4,428 \\
(Percent) & $(71.5)$ & $(12.1)$ & $(5.0)$ & $(4.7)$ & $(3.0)$ & $(2.1)$ & $(1.4)$ & $(0.2)$ & $(100.0)$ \\
McDonald's: Frequency & 3,638 & 364 & 102 & 128 & 80 & 62 & 50 & 4 & 4,428 \\
$\quad$ (Percent) & $(82.2)$ & $(8.2)$ & $(2.3)$ & $(2.9)$ & $(1.8)$ & $(1.4)$ & $(1.1)$ & $(0.1)$ & $(100.0)$ \\
\hline \hline Number of New Outlets & 0 & 1 & 2 & $3-5$ & $6-10$ & $11-20$ & $21-30$ & $>30$ & Total \\
(opened during the year) & & & & & & & & & \\
\hline KFC: Frequency & 3,643 & 454 & 140 & 128 & 37 & 18 & 7 & 1 & 4,428 \\
$\quad$ (Percent) & $(82.3)$ & $(10.3)$ & $(3.2)$ & $(2.9)$ & $(0.8)$ & $(0.4)$ & $(0.2)$ & $(0.0)$ & $(100.1)^{\S}$ \\
McDonald's: Frequency & 4,018 & 225 & 70 & 75 & 30 & 9 & 1 & 0 & 4,428 \\
$\quad$ Percent) & $(90.7)$ & $(5.1)$ & $(1.6)$ & $(1.7)$ & $(0.7)$ & $(0.2)$ & $(0.0)$ & $(0)$ & $(100.0)$ \\
\hline \hline
\end{tabular}

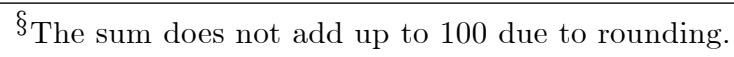


Table 5: Summary statistics

\begin{tabular}{|c|c|c|c|c|c|}
\hline Variable (units) & Obs. & Mean & S.D & $p_{10}$ & $p_{90}$ \\
\hline \multicolumn{6}{|c|}{ Chain Characteristics (KFC) } \\
\hline Total outlets (number at the start of the period) & 4428 & 1.8 & 8.8 & 0 & 3 \\
\hline New outlets (number per period) & 4428 & 0.4 & 1.7 & 0 & 1 \\
\hline Distance from nearest own outlet (kilometer) & 4428 & 327 & 442 & 0 & 972 \\
\hline Network density ${ }^{1}$ & 4428 & 10.8 & 24.0 & 0 & 29.2 \\
\hline \multicolumn{6}{|c|}{ Chain Characteristics (McDonald's) } \\
\hline Total outlets (number at the start of the period) & 4428 & 1.2 & 6.3 & 0 & 1 \\
\hline New outlets (number per period) & 4428 & 0.2 & 1.1 & 0 & 0 \\
\hline Distance from nearest own outlet (kilometer) & 4428 & 647 & 1,095 & 0 & 1,517 \\
\hline Network density ${ }^{1}$ & 4428 & 7.9 & 18.6 & 0 & 21.8 \\
\hline \multicolumn{6}{|c|}{ City Characteristics } \\
\hline Pop. Density (thousand per sq. km.) & 4263 & 1.21 & 1.12 & 0.23 & 2.55 \\
\hline GDP p.c. (2001 prices, thousand yuans) & 4042 & 14.8 & 15.8 & 3.7 & 29.3 \\
\hline
\end{tabular}

Table 6: Annual operating income (2001 prices, million yuans)

\begin{tabular}{lcc}
\hline \hline & KFC & McDonald's \\
\hline Per City & & \\
Mean & 328.57 & 83.04 \\
S. D. & 489.83 & 141.36 \\
Per Outlet & & \\
Mean & 17.91 & 9.26 \\
S. D. & 20.29 & 5.44 \\
Observations & 84 & 170 \\
\hline \hline
\end{tabular}


Table 7: Static profit functions

\begin{tabular}{lcc}
\hline & KFC & McDonald's \\
\hline Specification in $(3):$ & & \\
$\theta_{i 0}^{\pi}$ & $17.234^{* * *}$ & $10.228^{* * *}$ \\
$($ S.E. $)$ & $(1.567)$ & $(0.570)$ \\
$\theta_{i n_{i}}^{\pi}$ & $-0.129^{* * *}$ & $-0.022^{*}$ \\
& $(0.017)$ & $(0.013)$ \\
$\theta_{i n_{j}}^{\pi}$ & -0.022 & -0.005 \\
& $(0.023)$ & $(0.011)$ \\
$\theta_{i P}^{\pi}$ & $0.001^{* * *}$ & $-5.46 E-5$ \\
& $(0.000)$ & $(9.84 E-5)$ \\
$R^{2}$ & 0.901 & 0.893 \\
\hline Specification in $(4):$ & & \\
$\theta_{i f}^{\pi}$ & $4.524^{* * *}$ & $6.617^{* * *}$ \\
$($ S.E. $)$ & $(1.490)$ & $(0.000)$ \\
$\theta_{i P /\left(n_{i}+n_{j}\right)}^{\pi}$ & $0.141^{* * *}$ & $0.019^{* * *}$ \\
$R^{2}$ & $(0.024)$ & $(0.005)$ \\
\hline Observations & 0.861 & 0.886 \\
\hline \hline Note: ${ }^{* * *}{ }^{* *}$ and ${ }^{*}$ denote significance at $1 \%, 5 \%$ and \\
$10 \%$ levels, respectively. & & 170 \\
\hline
\end{tabular}


Table 8: First-stage policies

\begin{tabular}{|c|c|c|c|c|}
\hline \multirow[b]{2}{*}{ Variable } & \multicolumn{2}{|c|}{ KFC } & \multicolumn{2}{|c|}{ McDonald's } \\
\hline & Estimate & (S.E.) & Estimate & (S.E.) \\
\hline$n_{i}$ & $0.100^{* * *}$ & $(0.029)$ & $0.159^{* * *}$ & $(0.036)$ \\
\hline$n_{i}^{2}$ & $-1.15 E-3^{* * *}$ & $(1.7 E-4)$ & $-2.62 E-3$ & $(5.87 E-4)$ \\
\hline$n_{i}^{3}$ & $3.77 E-6^{* * *}$ & $(6.06 E-7)$ & $1.82 E-5$ & $(5.64 E-6)$ \\
\hline$n_{j}$ & $0.082^{* * *}$ & $(0.013)$ & $0.065^{* * *}$ & $(0.017)$ \\
\hline$n_{j} \times I_{i}$ & -0.017 & $(0.282)$ & $1.031^{* * *}$ & $(0.170)$ \\
\hline$n_{j}^{2}$ & $-1.72 E-3^{* * *}$ & $(3.36 E-4)$ & $-5.88 E-4^{*}$ & $(3.15 E-4)$ \\
\hline$n_{j}^{2} \times I_{i}$ & 0.035 & $(0.095)$ & $-0.166^{* * *}$ & $(0.032)$ \\
\hline$n_{j}^{3}$ & $1.25 E-5^{* * *}$ & $(3.08 E-6)$ & $3.17 E-6^{* * *}$ & $(1.06 E-6)$ \\
\hline$n_{j}^{3} \times I_{i}$ & -0.002 & $(0.007)$ & $0.007^{* * *}$ & $(0.001)$ \\
\hline$a_{i}$ & $0.299^{* * *}$ & $(0.067)$ & $0.198^{* *}$ & $(0.088)$ \\
\hline$a_{i} \times I_{i}$ & $-0.403^{* * *}$ & $(0.042)$ & $-0.420^{* * *}$ & $(0.063)$ \\
\hline$a_{i}^{2}$ & $-0.014^{* * *}$ & $(0.003)$ & $-0.017^{* * *}$ & $(0.004)$ \\
\hline$a_{i}^{2} \times I_{i}$ & $0.025^{* * *}$ & $(0.002)$ & $-0.023^{* * *}$ & $(0.004)$ \\
\hline$d_{i}$ & $-1.54 E-3^{* * *}$ & $(4.83 E-4)$ & $-1.77 E-3^{* * *}$ & $(4.11 E-4)$ \\
\hline$d_{j}$ & $2.77 E-4^{* *}$ & $(1.32 E-4)$ & $-1.14 E-4$ & $(6.43 E-4)$ \\
\hline$d_{j} \times I_{i}$ & $-8.01 E-4^{* * *}$ & $(2.50 E-4)$ & $-1.79 E-3^{* *}$ & $(8.46 E-4)$ \\
\hline$(n w)_{i}$ & $0.013^{* * *}$ & $(0.002)$ & -0.002 & $(0.004)$ \\
\hline$(n w)_{i} \times I_{i}$ & -0.010 & $(0.008)$ & $0.029^{* * *}$ & $(0.009)$ \\
\hline$(n w)_{j}$ & $-0.013^{* * *}$ & $(0.002)$ & -0.001 & $(0.003)$ \\
\hline$(n w)_{j} \times I_{i}$ & 0.013 & $(0.008)$ & $-0.014^{*}$ & $(0.007)$ \\
\hline Den & $0.118^{* * *}$ & $(0.032)$ & $0.164^{* *}$ & $(0.077)$ \\
\hline $\operatorname{Den} \times I_{i}$ & 0.044 & $(0.061)$ & -0.159 & $(0.098)$ \\
\hline$G D P p c$ & $0.008^{* * *}$ & $(0.002)$ & $0.010^{* *}$ & $(0.005)$ \\
\hline$G D P p c \times I_{i}$ & $0.016^{* * *}$ & $(0.006)$ & $0.016^{*}$ & $(0.008)$ \\
\hline$n_{i} \times n_{j}$ & $-2.51 E-4$ & $(2.98 E-4)$ & $-6.88 E-4$ & $(5.53 E-4)$ \\
\hline$n_{i} \times a_{i}$ & 0.002 & $(0.002)$ & $-7.22 E-4$ & $(0.003)$ \\
\hline$n_{i} \times(n w)_{i}$ & $-3.66 E-4^{* * *}$ & $(8.54 E-5)$ & $3.93 E-4$ & $(1.90 E-4)$ \\
\hline$n_{i} \times D e n$ & -0.003 & $(0.004)$ & -0.011 & $(0.009)$ \\
\hline$n_{i} \times G D P p c$ & $-4.40 E-5$ & $(-4.68 E-5)$ & $-2.28 E-4$ & $(1.19 E-4)$ \\
\hline over-dispersion $\chi^{2}$ ( $p$-value) & 92.45 & $(0.000)$ & 196.73 & $(0.000)$ \\
\hline$R^{2}$ & 0.3 & & 0.28 & \\
\hline Observations & 40 & & 404 & \\
\hline
\end{tabular}

Note: ${ }^{* * *},{ }^{* *}$ and ${ }^{*}$ denote significance at $1 \%, 5 \%$ and $10 \%$ levels, respectively. 
Table 9: Structural parameter estimates

\begin{tabular}{|c|c|c|}
\hline Parameters & KFC & $\mathrm{McD}$ \\
\hline $\begin{array}{c}\theta_{i 0}^{e} \\
(S . E .)\end{array}$ & $\begin{array}{c}416.55 \\
(1043.35)\end{array}$ & $\begin{array}{l}31.71^{* *} \\
(17.88)\end{array}$ \\
\hline$\theta_{i P / A}^{e}$ & $\begin{array}{c}346.15^{* * *} \\
(45.32)\end{array}$ & $\begin{array}{c}18.11^{* * *} \\
(2.72)\end{array}$ \\
\hline$\theta_{i y}^{e}$ & $\begin{array}{c}21.57^{* * *} \\
(8.30)\end{array}$ & $\begin{array}{c}1.07^{* * *} \\
(0.35)\end{array}$ \\
\hline$\theta_{i a_{i}}^{e}$ & $\begin{array}{c}76.23 \\
(118.24)\end{array}$ & $\begin{array}{c}-11.21^{* * *} \\
(2.66)\end{array}$ \\
\hline$\theta_{i a_{i}^{2}}^{e}$ & $\begin{array}{c}45.96^{* * *} \\
(7.62)\end{array}$ & $\begin{array}{c}0.49^{* * *} \\
(0.15)\end{array}$ \\
\hline$\theta_{i d_{i}}^{e}$ & $\begin{array}{c}-3.01^{* * *} \\
(0.42)\end{array}$ & $\begin{array}{c}-0.21^{* * *} \\
(0.02)\end{array}$ \\
\hline$\theta_{i d_{j}}^{e}$ & $\begin{array}{c}-1.80^{* * *} \\
(0.29)\end{array}$ & $\begin{array}{c}-0.62^{* * *} \\
(0.08)\end{array}$ \\
\hline$\theta_{i n_{j}}^{e}$ & $\begin{array}{c}-220.18^{* * *} \\
(36.88)\end{array}$ & $\begin{array}{c}24.24^{* * *} \\
(3.18)\end{array}$ \\
\hline$\sigma_{i}^{e}$ & $\begin{array}{c}-148.27^{* *} \\
(66.99) \\
\end{array}$ & $\begin{array}{c}40.74^{* * *} \\
(10.78) \\
\end{array}$ \\
\hline$\theta_{i 0}^{s}$ & $\begin{array}{c}-103.35^{* * *} \\
(21.88)\end{array}$ & $\begin{array}{c}-79.43^{* * *} \\
(1.34)\end{array}$ \\
\hline$\theta_{i P / A}^{s}$ & $\begin{array}{c}0.11 \\
(0.39)\end{array}$ & $\begin{array}{c}0.02 \\
(0.06)\end{array}$ \\
\hline$\theta_{i y}^{s}$ & $\begin{array}{c}0.74^{* * *} \\
(0.10)\end{array}$ & $\begin{array}{c}0.004 \\
(0.005)\end{array}$ \\
\hline$\theta_{i a_{i}}^{s}$ & $\begin{array}{l}-2.78 \\
(2.54)\end{array}$ & $\begin{array}{c}-0.54^{* * *} \\
(0.13)\end{array}$ \\
\hline \multirow[t]{2}{*}{$\theta_{i a_{i}^{2}}^{s}$} & 0.03 & $0.005^{*}$ \\
\hline & $(0.07)$ & $(0.004)$ \\
\hline$\theta_{i w_{i}}^{s}$ & $\begin{array}{c}-0.17^{* * *} \\
(0.05)\end{array}$ & $\begin{array}{c}0.05^{* * *} \\
(0.01)\end{array}$ \\
\hline$\theta_{i w_{j}}^{s}$ & $\begin{array}{l}0.07^{*} \\
(0.05)\end{array}$ & $\begin{array}{c}-0.03^{* * *} \\
(0.004)\end{array}$ \\
\hline$\theta_{i x_{i}}^{s}$ & $\begin{array}{c}-1.35^{* * *} \\
(0.36)\end{array}$ & $\begin{array}{c}-0.72^{* * *} \\
(0.08)\end{array}$ \\
\hline$\theta_{i n_{i}}^{s}$ & $\begin{array}{c}1.60^{* * * *} \\
(0.11)\end{array}$ & $\begin{array}{c}0.39^{* * *} \\
(0.01)\end{array}$ \\
\hline$\theta_{i n_{j}}^{s}$ & $\begin{array}{l}0.20^{* *} \\
(0.09)\end{array}$ & $\begin{array}{l}0.06^{* * *} \\
(0.004)\end{array}$ \\
\hline$\sigma_{i}^{s}$ & $\begin{array}{l}22.78^{* *} \\
(12.99)\end{array}$ & $\begin{array}{c}-7.97^{* * *} \\
(1.72)\end{array}$ \\
\hline$\% \mathrm{CS}^{1}$ & 95.06 & 93.29 \\
\hline
\end{tabular}


Table 10: Spillovers

\begin{tabular}{lcc}
\hline \hline & KFC & McDonald's \\
\cline { 2 - 3 } Cause: & \multicolumn{2}{c}{$\begin{array}{c}\text { Change in Entry Cost } \\
\text { (in units of SD) }\end{array}$} \\
\cline { 2 - 3 } 100-kilometer decrease in rival's distance: & -1.22 & -1.52 \\
100-kilometer decrease in own distance: & -2.03 & -0.51 \\
One-unit increase in rival's outlets: & 1.49 & -0.59 \\
\hline & \multicolumn{2}{c}{ Change in Sunk Cost } \\
Cause: & \multicolumn{2}{c}{ (in units of SD) } \\
\cline { 2 - 3 } 10-unit increase in rival's network density: & -0.03 & 0.03 \\
10-unit increase in own network density: & 0.07 & -0.06 \\
One-unit increase in rival's outlets: & -0.01 & -0.01 \\
One-unit increase in own outlets: & -0.07 & -0.05 \\
\hline \hline
\end{tabular}

${ }^{1} 10$-unit increase is equal to 10 more outlets in the city or 20 more outlets in a city $150 \mathrm{~km}$ away. See (1) for the exact definition of network density. 


\section{A Data on Operating Income}

We have compiled the data on operating income from a number of sources. The data for the years 2003-2005 are from Statistical Yearbook of China Restaurants (Issues 2004, 2005 and 2006). The data for years 2006 and 2007 are from Statistical Yearbook of China Chain Stores of Retail Trades and Catering Services (Issues 2007 and 2008). Instead of reporting a precise number for the operating income, the yearbooks report a range. We take the average of the lower and upper limits of the range as our measure of operating income. We expand our dataset by using information from the following online sources:

1. http://www.docin.com/p-69630763.html

2. http://www . baiyan.cc/newsInfo.asp?typenumber=0002\&id=106

3. http://news.sohu.com/20060902/n245129062.shtml

4. http://finance.qq.com/a/20090327/000223.htm

5. http://www.canyin168.com/glyy/glzx/hyfx/201103/28410.html

We convert the data into constant 2001 yuans by using the Consumer's Price Index.

Our dataset on operating income primarily covers big cities. For example, in our full sample the average number of outlets per city for the 2003-2007 period is 4.9 for KFC and 2.9 for McDonald's. If we restrict the sample to cities for which we have data on operating income, the average number of outlets per city becomes 21.3 for KFC and 9.3 for McDonald's. Consequently, the static profits that we estimate may not be representative of the average profits in the entire country. One way to see this is to use country-level sales figures for the two chains, assume a reasonable mark-up and compute country-level profits. We can then compare them with the country-level profits implied by our estimates. According to The Wall Street Journal (2013) China's fastfood sales were $\$ 47$ billion in 2007. The market shares of KFC and McDonald's were $6.5 \%$ and $2.3 \%$, respectively. This leads to the sales of about $\$ 3.055$ billion for KFC and $\$ 1.081$ billion for McDonald's. If one assumes a markup of $30 \%$ on cost, the implied profits are $\$ 0.705$ billion for KFC and $\$ 0.249$ billion for McDonald’s. We estimate average profit per outlet to be ¥17.234 million for KFC and ¥10.228 million for McDonald’s. The exchange rate between yuan and dollar was 7.5910 yuan per dollar on July 2, 2007 (Source: www.finance.yahoo.com). Using this exchange rate our estimated average profit per outlet works out to be $\$ 2.270$ million for KFC and $\$ 1.347$ million for McDonald's. These translate into country-level estimated profits of $\$ 4.386$ billion $(=\$ 2.270 \mathrm{~m} \times 1932$ outlets $)$ for KFC and $\$ 1.363$ billion $(=\$ 1.347 \mathrm{~m} \times 1012$ outlets $)$. These simple back-of-the-envelope calculations show that we might be overestimating the static profits by a factor of $6.2(4.386 / 0.705)$ for KFC and by a factor of $5.5(1.363 / 0.249)$ for McDonald's. We discuss the implications of this over-estimation in Section 6.3 


\section{B Appendix Figures}

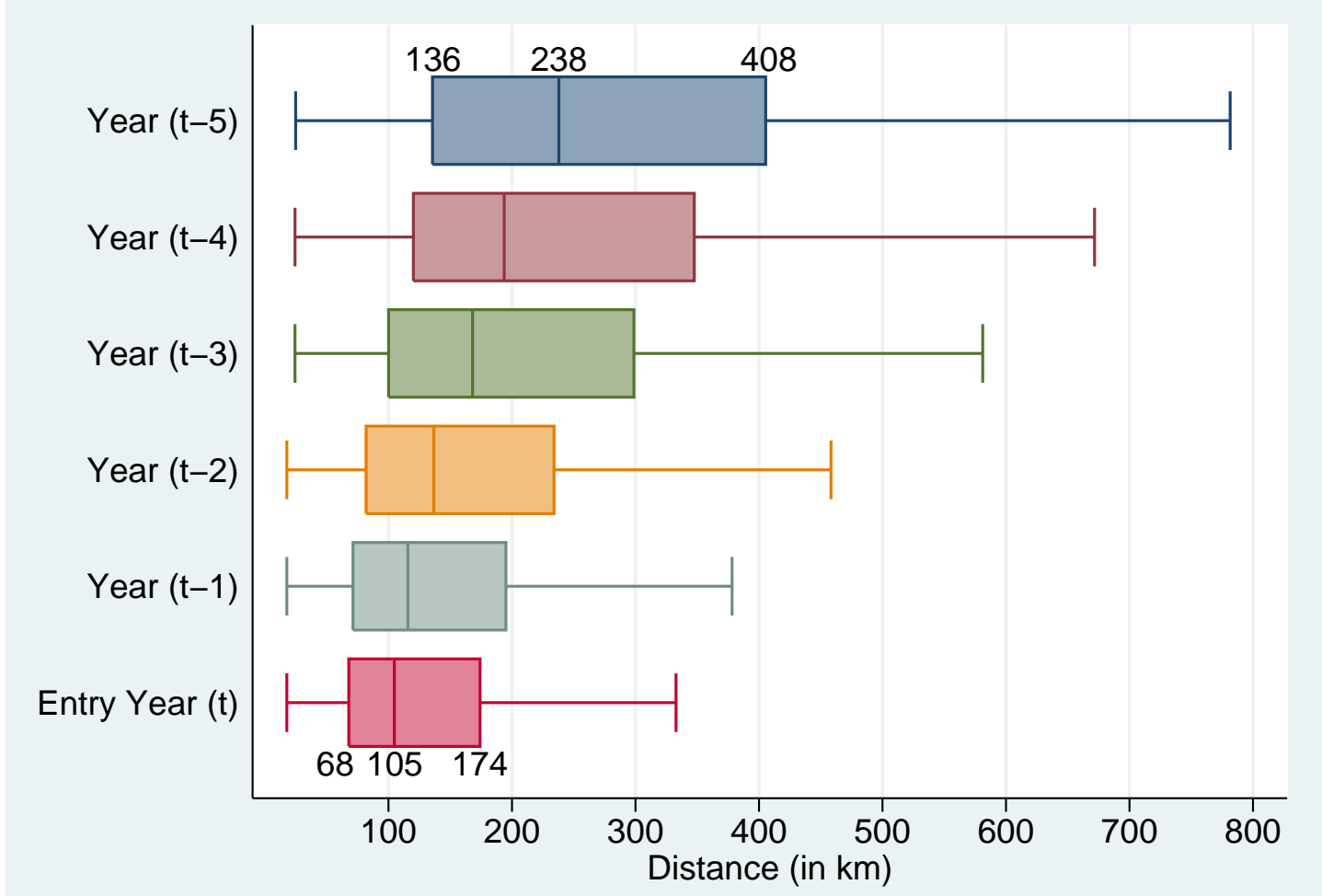

Figure B.1: Distance from the nearest city with at least one own outlet (KFC) 


\section{Appendix Tables}

Table C.1: Discretization of the distance variable

\begin{tabular}{ccc}
\hline \hline State & Range in the data $(\mathrm{km})$ & Discretized value \\
\hline 1 & 0 & 0 \\
2 & $0<d \leq 50$ & 25 \\
3 & $50<d \leq 100$ & 75 \\
4 & $100<d \leq 150$ & 125 \\
5 & $150<d \leq 200$ & 175 \\
6 & $200<d \leq 300$ & 250 \\
7 & $300<d \leq 500$ & 400 \\
8 & $500<d \leq 1,000$ & 750 \\
9 & $1,000<d \leq 2,000$ & 1,500 \\
10 & $2,000<d \leq 3,000$ & 2,500 \\
11 & $3,000<d \leq 4,000$ & 3,500 \\
12 & $4,000<d \leq 5,000$ & 4,500 \\
\hline \hline
\end{tabular}


Table C.2: Estimated transition matrix for GDP p.c. (thousand yuans)

\begin{tabular}{|c|c|c|c|c|c|c|c|c|}
\hline \hline Current $\backslash$ Next & $0-4$ & $4-8$ & $8-12$ & $12-16$ & $16-20$ & $20-30$ & $30-50$ & $>50$ \\
\hline $0-4$ & $\mathbf{0 . 6 9}$ & 0.29 & 0.01 & 0.00 & & & & \\
\hline $4-8$ & 0.02 & $\mathbf{0 . 7 5}$ & 0.21 & 0.02 & 0.00 & 0.00 & 0.00 & \\
\hline $8-12$ & 0.00 & 0.03 & $\mathbf{0 . 7 2}$ & 0.24 & 0.01 & 0.00 & 0.00 & \\
\hline $12-16$ & 0.00 & 0.00 & 0.03 & $\mathbf{0 . 6 4}$ & 0.31 & 0.02 & & \\
\hline $16-20$ & & 0.00 & 0.01 & 0.05 & $\mathbf{0 . 5 3}$ & 0.40 & 0.00 & 0.00 \\
\hline $20-30$ & & & 0.01 & 0.02 & 0.03 & $\mathbf{0 . 7 4}$ & 0.21 & \\
\hline $30-50$ & & & & & 0.00 & 0.02 & $\mathbf{0 . 8 3}$ & 0.14 \\
\hline$>50$ & & & & & & & 0.05 & $\mathbf{0 . 9 5}$ \\
\hline \hline
\end{tabular}

Note: Row sums may not be equal to 1 due to rounding.

Table C.3: Estimated transition matrix for population (thousands)

\begin{tabular}{|c|c|c|c|c|c|c|c|}
\hline \hline Current $\backslash$ Next & $0-250$ & $250-500$ & $500-750$ & $750-1000$ & $1000-1500$ & $1500-2000$ & $>2000$ \\
\hline $0-250$ & $\mathbf{0 . 8 1}$ & 0.17 & 0.01 & & & 0.01 & \\
\hline $250-500$ & 0.00 & $\mathbf{0 . 9 4}$ & 0.04 & 0.00 & 0.01 & 0.01 & 0.00 \\
\hline $500-750$ & 0.00 & 0.01 & $\mathbf{0 . 9 3}$ & 0.04 & 0.01 & 0.00 & 0.00 \\
\hline $750-1000$ & & 0.00 & 0.01 & $\mathbf{0 . 9 4}$ & 0.04 & & 0.00 \\
\hline $1000-1500$ & 0.00 & 0.00 & 0.01 & 0.01 & $\mathbf{0 . 9 4}$ & 0.04 & 0.01 \\
\hline $1500-2000$ & & 0.01 & & & 0.01 & $\mathbf{0 . 9 3}$ & 0.04 \\
\hline$>2000$ & & & & & & 0.00 & $\mathbf{1 . 0 0}$ \\
\hline \hline
\end{tabular}

Note: Row sums may not be equal to 1 due to rounding. 
Table C.4: Estimated transition matrix for distance (KFC) in kilometers

\begin{tabular}{|c|c|c|c|c|c|c|c|c|c|c|c|c|}
\hline \hline$t \backslash(t+1)$ & 0 & $0-50$ & $50-100$ & $100-150$ & $150-200$ & $200-300$ & $300-500$ & $500-1 \mathrm{~K}$ & $1 \mathrm{~K}-2 \mathrm{~K} 2 \mathrm{~K}-3 \mathrm{~K} 3 \mathrm{~K}-4 \mathrm{~K} 4 \mathrm{~K}-5 \mathrm{~K}$ \\
\hline 0 & 1.00 & & & & & & & & & & & \\
\hline $0-50$ & 0.21 & 0.79 & & & & & & & & & & \\
\hline $50-100$ & 0.17 & 0.03 & 0.80 & & & & & & & & & \\
\hline $100-150$ & 0.15 & 0.02 & 0.08 & 0.75 & & & & & & & & \\
\hline $150-200$ & 0.08 & 0.00 & 0.06 & 0.12 & 0.73 & & & & & & & \\
\hline $200-300$ & 0.05 & 0.01 & 0.05 & 0.07 & 0.11 & 0.71 & & & & & & \\
\hline $300-500$ & 0.03 & 0.00 & 0.03 & 0.03 & 0.03 & 0.10 & 0.78 & & & & & \\
\hline $500-1 \mathrm{~K}$ & 0.01 & 0.00 & 0.02 & 0.01 & 0.02 & 0.05 & 0.15 & 0.74 & & & & \\
\hline $1 \mathrm{~K}-2 \mathrm{~K}$ & 0.01 & 0.00 & 0.02 & 0.01 & 0.01 & 0.01 & 0.03 & 0.16 & 0.75 & & & \\
\hline $2 \mathrm{~K}-3 \mathrm{~K}$ & & & & & & & 0.03 & & 0.13 & 0.84 & & \\
\hline $3 \mathrm{~K}-4 \mathrm{~K}$ & & & & & & & & & & 0.25 & 0.75 & \\
\hline $4 \mathrm{~K}-5 \mathrm{~K}$ & 0.00 & & 0.02 & 0.02 & 0.00 & 0.03 & 0.06 & 0.24 & 0.48 & 0.13 & 0.01 & \\
\hline \hline
\end{tabular}

Note: Row sums may not be equal to 1 due to rounding.

Table C.5: Estimated transition matrix for distance (McDonald's) in kilometers

\begin{tabular}{|c|c|c|c|c|c|c|c|c|c|c|c|c|c|}
\hline \hline$t \backslash(t+1)$ & 0 & $0-50$ & $50-100$ & $100-150150-200$ & $200-300300-500$ & $500-1 \mathrm{~K} 1 \mathrm{~K}-2 \mathrm{~K} 2 \mathrm{~K}-3 \mathrm{~K} 3 \mathrm{~K}-4 \mathrm{~K} 4 \mathrm{~K}-5 \mathrm{~K}$ \\
\hline 0 & 1.00 & & & & & & & & & & & \\
\hline $0-50$ & 0.15 & 0.85 & & & & & & & & & & \\
\hline $50-100$ & 0.08 & 0.03 & 0.89 & & & & & & & & & \\
\hline $100-150$ & 0.08 & 0.01 & 0.07 & 0.84 & & & & & & & & \\
\hline $150-200$ & 0.04 & 0.01 & 0.06 & 0.11 & 0.78 & & & & & & & \\
\hline $200-300$ & 0.04 & 0.01 & 0.05 & 0.08 & 0.09 & 0.74 & & & & & & \\
\hline $300-500$ & 0.03 & 0.01 & 0.01 & 0.03 & 0.04 & 0.11 & 0.78 & & & & & \\
\hline $500-1 \mathrm{~K}$ & 0.01 & 0.00 & 0.02 & 0.02 & 0.03 & 0.05 & 0.12 & 0.76 & & & & \\
\hline $1 \mathrm{~K}-2 \mathrm{~K}$ & 0.00 & 0.00 & 0.00 & 0.00 & 0.01 & 0.01 & 0.07 & 0.23 & 0.68 & & & \\
\hline $2 \mathrm{~K}-3 \mathrm{~K}$ & & & & & & & 0.02 & 0.19 & 0.16 & 0.62 & & \\
\hline $3 \mathrm{~K}-4 \mathrm{~K}$ & & & & & & & & & 0.08 & 0.15 & 0.77 & \\
\hline $4 \mathrm{~K}-5 \mathrm{~K}$ & 0.00 & & 0.02 & 0.02 & 0.00 & 0.03 & 0.06 & 0.24 & 0.48 & 0.13 & 0.01 & \\
\hline \hline
\end{tabular}

Note: Row sums may not be equal to 1 due to rounding. 
Table C.6: Estimated transition matrix for network density (KFC)

\begin{tabular}{|c|c|c|c|c|c|c|c|c|c|c|c|c|}
\hline \hline$t \backslash(t+1)$ & 0 & $0-1$ & $1-2$ & $2-3$ & $3-5$ & $5-10$ & $10-15$ & $15-20$ & $20-30$ & $30-50$ & $50-100$ & $>100$ \\
\hline 0 & 0.87 & 0.11 & 0.02 & 0.00 & 0.00 & & & & & & & \\
\hline $0-1$ & & 0.61 & 0.29 & 0.08 & 0.02 & & & & & & & \\
\hline $1-2$ & & & 0.34 & 0.42 & 0.20 & 0.04 & & & & & & \\
\hline $2-3$ & & & & 0.22 & 0.67 & 0.10 & & & & & & \\
\hline $3-5$ & & & & & 0.35 & 0.63 & 0.01 & & & & & \\
\hline $5-10$ & & & & & & 0.50 & 0.46 & 0.04 & 0.00 & & & \\
\hline $10-15$ & & & & & & & 0.36 & 0.58 & 0.05 & & & \\
\hline $15-20$ & & & & & & & & 0.30 & 0.69 & 0.01 & & \\
\hline $20-30$ & & & & & & & & & 0.51 & 0.49 & & \\
\hline $30-50$ & & & & & & & & & & 0.60 & 0.40 & \\
\hline $50-100$ & & & & & & & & & & & 0.77 & 0.23 \\
\hline$>100$ & & & & & & & & & & & & 1.00 \\
\hline \hline
\end{tabular}

Note: Row sums may not be equal to 1 due to rounding.

Table C.7: Estimated transition matrix for network density (McDonald's)

\begin{tabular}{|c|c|c|c|c|c|c|c|c|c|c|c|c|}
\hline \hline$t \backslash(t+1)$ & 0 & $0-1$ & $1-2$ & $2-3$ & $3-5$ & $5-10$ & $10-15$ & $15-20$ & $20-30$ & $30-50$ & $50-100$ & $>100$ \\
\hline 0 & 0.88 & 0.09 & 0.01 & 0.01 & 0.00 & & & & & & & \\
\hline $0-1$ & & 0.60 & 0.22 & 0.10 & 0.06 & 0.01 & & & & & & \\
\hline $1-2$ & & & 0.36 & 0.31 & 0.22 & 0.11 & & & & & & \\
\hline $2-3$ & & & & 0.29 & 0.57 & 0.13 & 0.01 & & & & & \\
\hline $3-5$ & & & & & 0.43 & 0.51 & 0.04 & 0.01 & 0.00 & & & \\
\hline $5-10$ & & & & & & 0.68 & 0.28 & 0.02 & 0.01 & 0.01 & & \\
\hline $10-15$ & & & & & & & 0.59 & 0.36 & 0.04 & 0.01 & & \\
\hline $15-20$ & & & & & & & & 0.55 & 0.45 & & & \\
\hline $20-30$ & & & & & & & & & 0.62 & 0.38 & & \\
\hline $30-50$ & & & & & & & & & & 0.73 & 0.27 & \\
\hline $50-100$ & & & & & & & & & & & 0.88 & 0.12 \\
\hline$>100$ & & & & & & & & & & & & 1.00 \\
\hline \hline
\end{tabular}

Note: Row sums may not be equal to 1 due to rounding. 


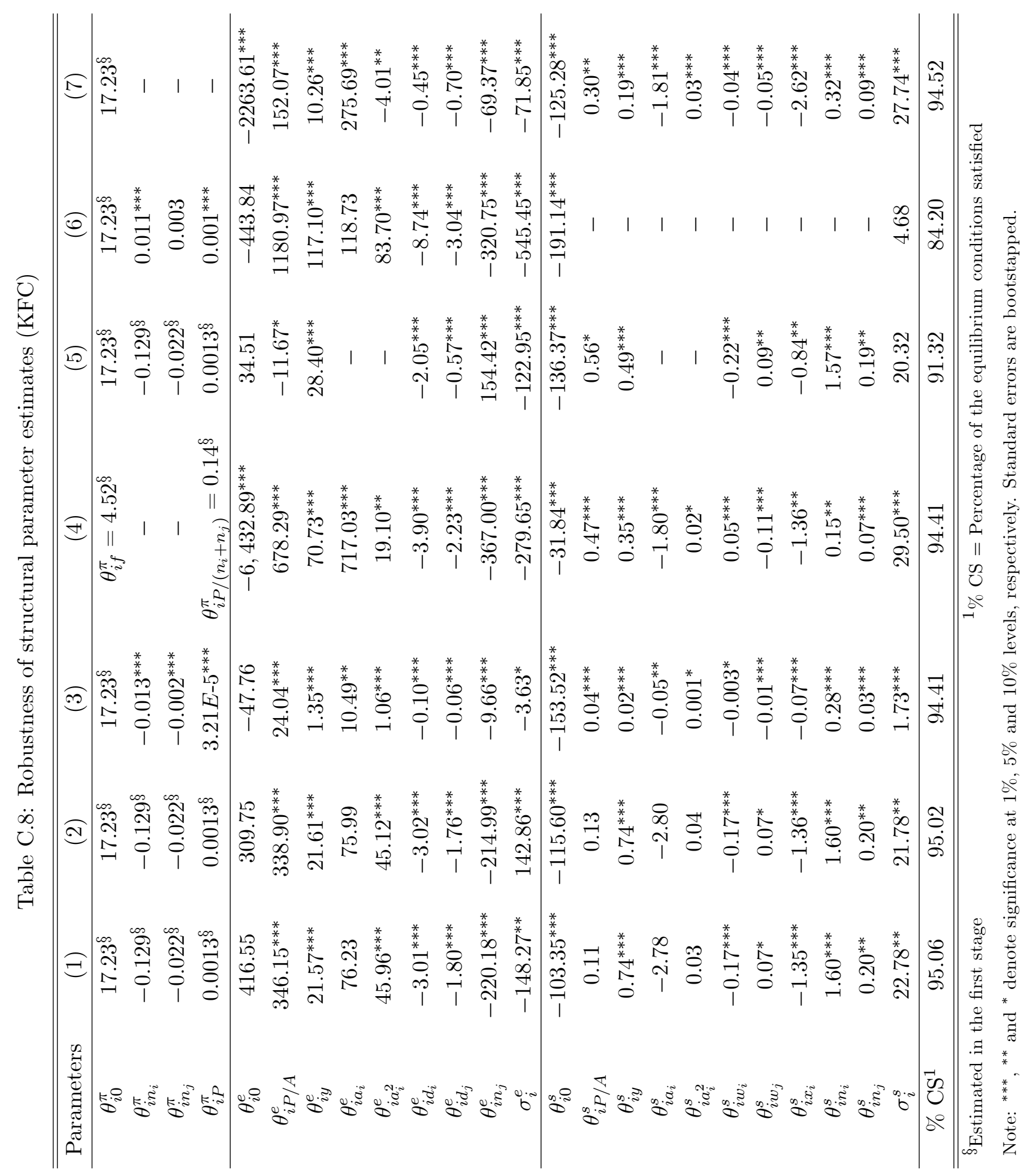




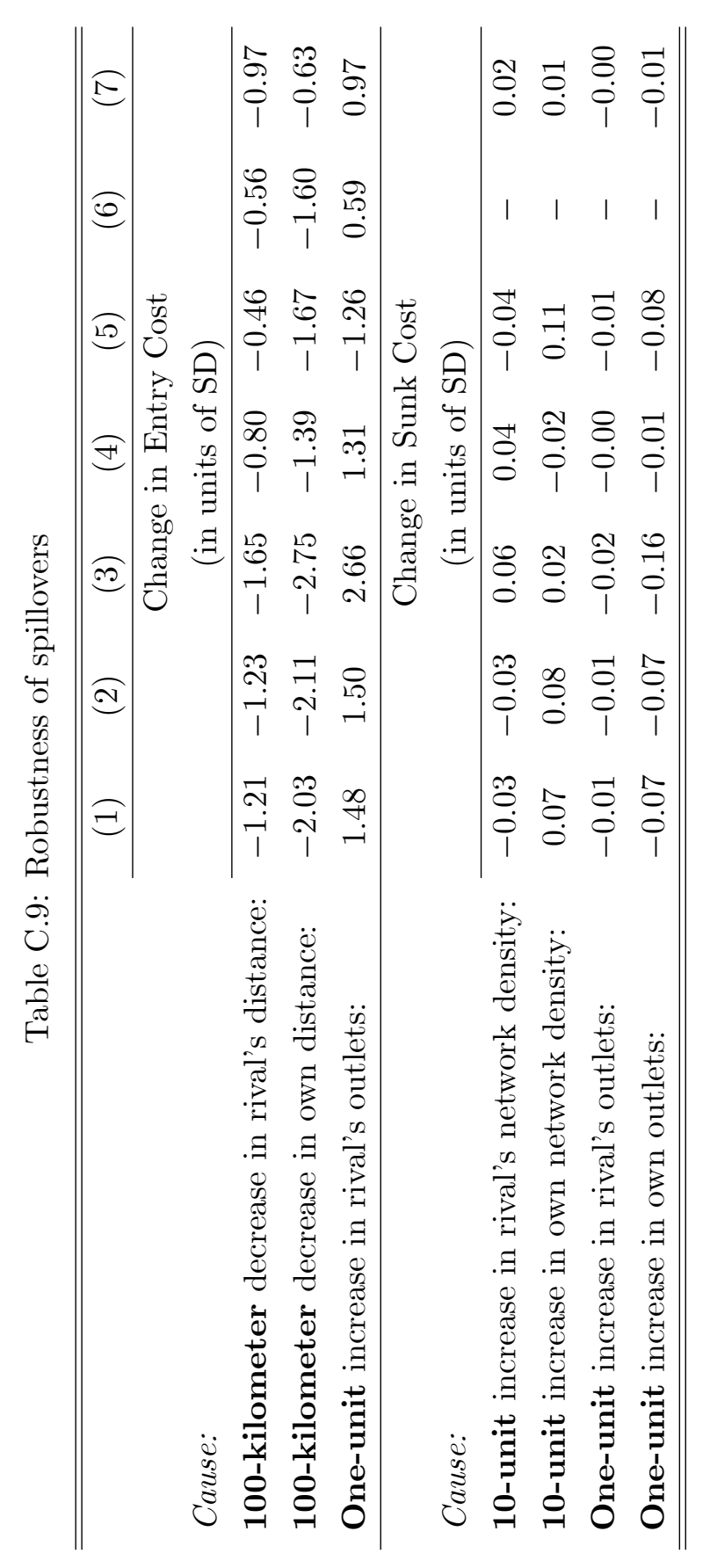




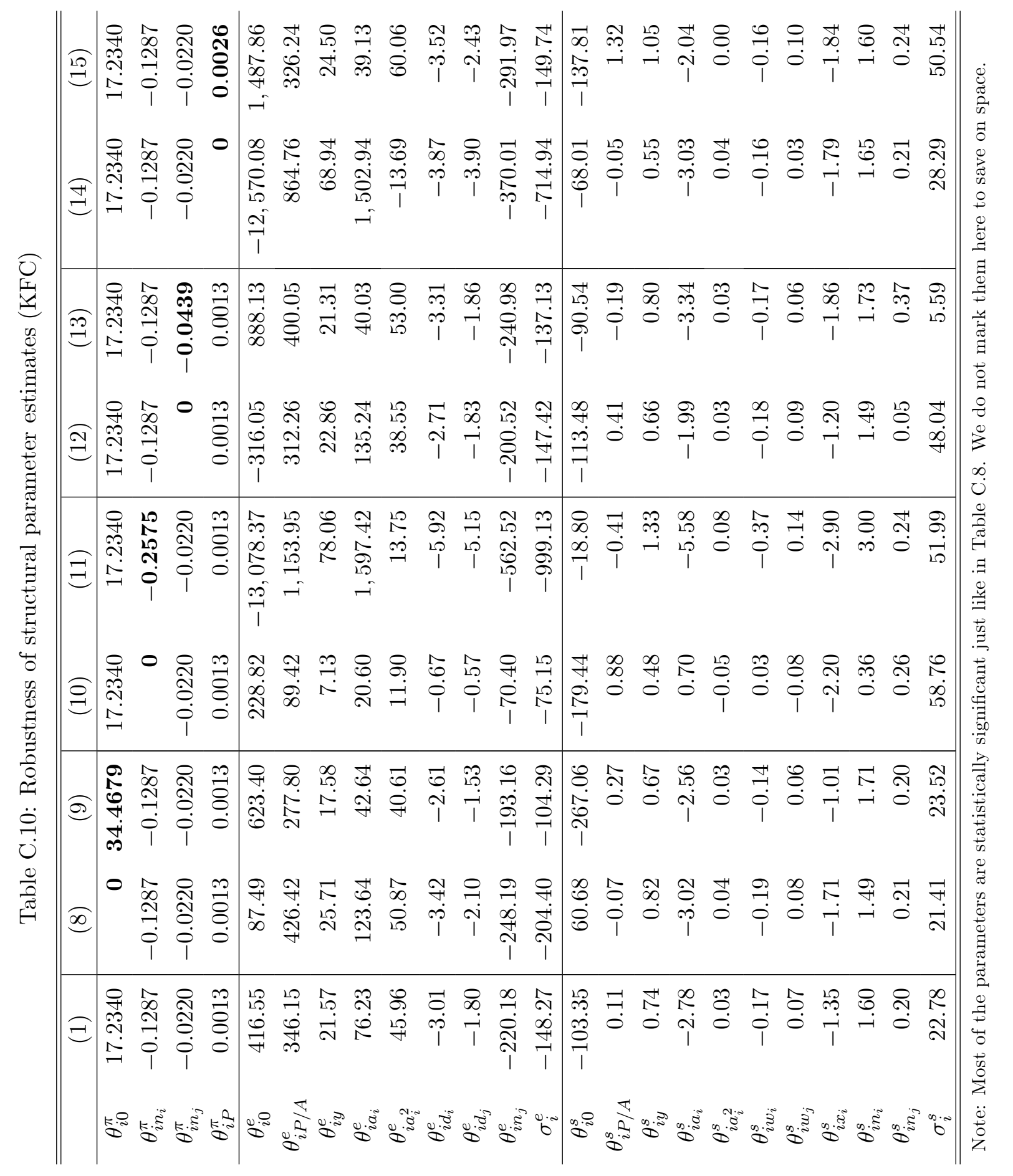




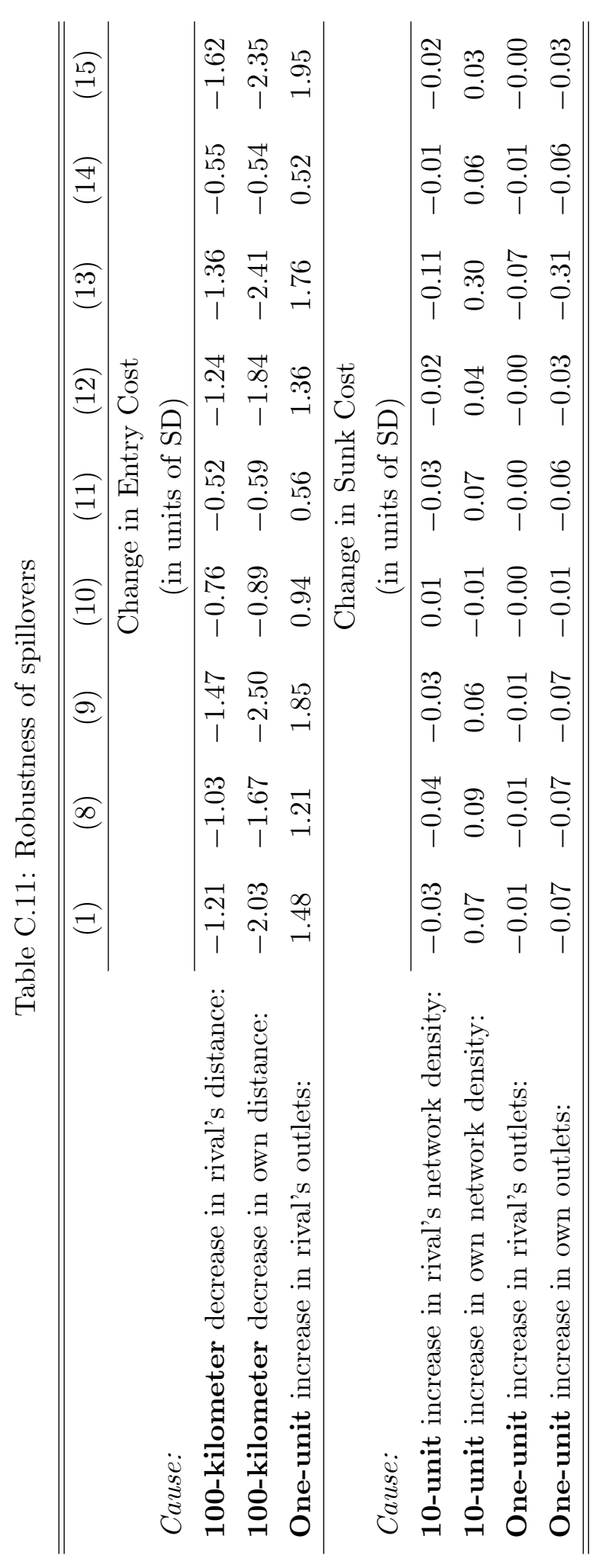

